\title{
BALANCED BASKETS: A NEW APPROACH TO TRADING AND HEDGING RISKS
}

\author{
David H. Bailey \\ Complex Systems Group Leader - Lawrence Berkeley National Laboratory \\ dhbailey@1bl.gov \\ Marcos López de Prado \\ Head of Global Quantitative Research - Tudor Investment Corporation \\ and \\ Research Affiliate - Lawrence Berkeley National Laboratory \\ lopezdeprado@lbl.gov
}

The Journal of Investment Strategies, Fall 2012

http://www.risk.net/type/journal/source/journal-of-investment-strategies

We are grateful to Tudor Investment Corporation, Robert Almgren (Quantitative Brokers, NYU), José Blanco
(UBS), Sid Browne (Guggenheim Partners), Peter Carr (Morgan Stanley, NYU), David Easley (Cornell Univ.),
Matthew Foreman (Univ. of California, Irvine), Ross Garon (S.A.C. Capital Advisors), Paul Glasserman (Columbia
Univ.), Robert Jarrow (Cornell Univ.), David Leinweber (Lawrence Berkeley National Laboratory), Yin Luo
(Deutsche Bank), Attilio Meucci (Kepos Capital, NYU), Maureen O'Hara (Cornell Univ.), Riccardo Rebonato
(PIMCO, Univ. of Oxford), Luis Viceira (HBS), as well as two anonymous referees.

Supported in part by the Director, Office of Computational and Technology Research, Division of Mathematical, Information, and Computational Sciences of the U.S. Department of Energy, under contract number DE-AC02$05 \mathrm{CH} 11231$. 


\title{
BALANCED BASKETS: A NEW APPROACH TO TRADING AND HEDGING RISKS
}

\begin{abstract}
A basket is a set of instruments that are held together because its statistical profile delivers a desired goal, such as hedging or trading, which cannot be achieved through the individual constituents or even subsets of them. Multiple procedures have been proposed to compute hedging and trading baskets, among which balanced baskets have attracted significant attention in recent years. Unlike Principal Component Analysis (PCA) style of methods, balanced baskets spread risk or exposure across their constituents without requiring a change of basis. Practitioners typically prefer balanced baskets because their output can be understood in the same terms for which they have developed an intuition.

We review three methodologies for determining balanced baskets, analyze the features of their respective solutions and provide Python code for their calculation. We also introduce a new method for reducing the dimension of a covariance matrix, called Covariance Clustering, which addresses the problem of numerical ill-conditioning without requiring a change of basis.
\end{abstract}

Keywords: Trading baskets, hedging baskets, equal risk contribution, maximum diversification, subset correlation.

JEL Classifications: C01, C02, C61, D53, G11. 


\section{INTRODUCTION}

A basket is a set of instruments that are held together because its statistical profile delivers a desired goal, such as hedging a risk or trading it, which cannot be achieved through the individual constituents or even subsets of them. Portfolio managers build trading baskets that translate their views of the markets into actual financial bets, while hedging their exposure to other risks they have no view on. Market makers build hedging baskets that allow them to offset the risk derived from undesired inventory. Quantitative researchers form hedging baskets as a mean to study, replicate or reverse-engineer the factors driving the performance of a security, portfolio or hedge fund (Jaeger (2008)).

Multiple procedures have been proposed to compute hedging and trading baskets. Balanced baskets have attracted significant attention in recent years because, unlike PCA-style methods (see Litterman and Scheinkman (1991), Moulton and Seydoux (1998), for example), they spread risk or exposure across its constituents without requiring a change of basis. A change of basis is problematic because the basket's solution is expressed in terms of the new basis (a linear combination of tradable instruments), which may not be intuitive in terms of the old basis. Practitioners typically prefer balanced baskets for this reason.

In this paper we will differentiate between the goal of hedging and the goal of trading. In the first instance, the basket is formed to reduce the investor's risk or exposure to any of its legs, or any subset of them. In the second instance, the investor would like to acquire risk or exposure to each and every of its legs (or subsets of them) in a balanced way. Although hedging baskets may appear to be the opposite of trading baskets, both concepts are intimately related and both can be computed using similar procedures.

López de Prado and Leinweber (2012) reviewed the literature on hedging methods. Among the methods they studied are Equal-Risk Contribution (ERC), Maximum Diversification Ratio (MDR) and Mini-Max Subset Correlation (MMSC). The three are static (time-invariant) methods that attempt to balance the risk or exposure of the basket among its constituents. MDR solves a "hedging" problem, while MMSC and ERC can be applied to solve both, a "hedging" as well as a "trading" problem.

Maillard, Roncalli and Teiletche (2009) and Demey, Maillard and Roncalli (2010) gave a formal definition of ERC. Previous descriptions can be found in Qian $(2005,2006)$ under the term "risk parity", Booth and Fama (1992), and earlier authors. This procedure attempts to balance the contribution of risk for each of the basket's legs. Empirical studies of ERC's performance against alternative weighting schemes can be found in Neukirch (2008), DeMiguel, Garlappi and Uppal (2009) and Hurst, Johnson and Ooi (2010). Most authors impose the constraints that all weights must be positive and add up to one, because they have in mind an asset allocation application. Our analysis is free of such constraints because we would like to discuss the problem of constructing a basket in general terms, rather than focusing on a particular use. This concept's popularity is illustrated by the many institutional asset managers offering ERC-weighted funds: PanAgora Asset Management, Bridgewater Associates, AQR Capital, Aquila Capital, Invesco, First Quadrant, Putnam Investments, ATP, Barclays Global Investors, Mellon Capital Management, State Street Global Advisors, ... to cite only a few. 
MDR was proposed by Choueifaty and Coignard (2008) and Choueifaty, Froidure and Reynier (2011). Their goal is to maximize a "diversification ratio" that effectively balances the basket's exposure to each leg, measured in terms of correlation. Like in the ERC case, these authors also incorporate constraints characteristic of an asset allocation framework, which we will obviate in this study for the sake of generality.

MMSC was introduced by López de Prado and Leinweber (2012). This procedure balances the exposure of the basket, not only to each leg (like MDR) but also to any subset of legs. The motivation is to reduce the basket's vulnerability to structural breaks, i.e. when a subset receives a shock that does not impact the rest of the basket. In a basket of two instruments, MMSC coincides with MDR, since the only subsets are the legs themselves. Furthermore, we will see that when only two instruments are considered, ERC, MDR and MMSC give the same solution. However, the three procedures exhibit substantial differences whenever we are dealing with baskets of more than two instruments.

The three procedures are theoretically sound. The purpose of this study is not to invalidate or criticize any of them, but to evidence the differences and properties associated with each solution. A second goal of this paper is to provide efficient algorithms for the calculation of ERC, MDR and MMSC. Hundreds of billions of dollars are invested using balanced basket approaches (particularly ERC), and yet no optimization algorithm can be found in the academic literature. A third, ancillary goal, is to provide a procedure for reducing the dimension of a covariance matrix to a number that makes these methodologies computationally feasible. We believe that our Covariance Clustering method has important applications for the management of risks in large portfolios of highly correlated instruments or funds. Given the analytical and algorithmic nature of this paper, an empirical study of the absolute and relative performance of balanced baskets over the past years is beyond its scope. Such study would merit an extensive and monographic discussion.

The rest of the paper is organized as follows: Section 2 discusses the hedging problem in a twodimensional framework. Section 3 evidences the qualitative difference between working in two dimensions and dealing with three or more. Section 4 extends our "hedging" analysis to the problem of computing "trading baskets." Section 5 summarizes our conclusions. Appendix 1 derives a numerical procedure for the calculation of ERC baskets. Appendix 2 presents a codification of that algorithm in Python. Appendices 3 and 4 do the same in the context of MMSC and MDR baskets. Appendix 5 describes the Covariance Clustering method, and includes its implementation in Python.

\section{THE TWO-DIMENSIONAL HEDGING PROBLEM}

Suppose that a portfolio manager wishes to hedge her position of 1,000 S\&P Midcap 400 E-mini futures contracts (Bloomberg code "FA1 Index") using S\&P 500 E-mini futures (Bloomberg code "ES1 Index"). The relevant covariance and correlation matrices on daily market value dollar changes $\left(\Delta P_{i}\right)$ are shown in Figure 1. As expected, we can appreciate a high and positive codependence between these two products, with an estimated correlation coefficient approaching 0.95 . 


\section{[FIGURE 1 HERE]}

Given some holdings $\omega$ and a covariance matrix $V$ of market value dollar changes, we can compute the variance of market value changes of the resulting basket $B$ of $n$ constituents as

$$
\sigma_{\Delta B}^{2}=\omega^{\prime} V \omega=\sum_{i=1}^{n} \sum_{j=1}^{n} \omega_{i} \sigma_{i, j} \omega_{j}
$$

where $\sigma_{i, j}$ is the covariance between $\Delta P_{i}$ and $\Delta P_{j}$. One option would be to compute the vector $\omega_{M V}$ that minimizes the basket's variance,

$$
\omega_{M V}=\arg \min _{\substack{\omega \\ \omega_{1}=h_{1}}} \sigma_{\Delta B}^{2}
$$

for which a general solution can be found in López de Prado and Leinweber (2012). In our particular case, $n=2$ and our holdings of "FA1 Index" are set fix to be $h_{1}=1,000$, so the only free holding is $\omega_{2}$. Deriving,

$$
\begin{gathered}
\frac{\partial \sigma_{\Delta B}^{2}}{\partial \omega_{2}}=2 h_{1} \sigma_{1,2}+2 \omega_{2} \sigma_{2}^{2} \\
\frac{\partial^{2} \sigma_{\Delta B}^{2}}{\partial \omega_{2}^{2}}=2 \sigma_{2}^{2}>0
\end{gathered}
$$

Because $\frac{\partial^{2} \sigma_{\Delta B}^{2}}{\partial \omega_{2}^{2}}>0$, applying the first order condition $\left(\frac{\partial \sigma_{\Delta B}^{2}}{\partial \omega_{2}}=0\right)$ will lead to a minimum at the point $\omega_{M V}=h_{1}\left(1,-\frac{\sigma_{1,2}}{\sigma_{2}^{2}}\right) \approx(1000,-1467.85)$, with $\sigma_{\Delta B} \approx 299,709.02$. The solution matches the coefficients of an Ordinary Least Squares (OLS) regression, which should have been expected, since the regression's objective function coincides with Eq. (2) for the case $n=2$.

Scherer (2010) and Clarke, de Silva and Thorley (2011) offer practical examples of OLS and Minimum Variance (MV) solutions. Although ubiquitous, the solutions provided by these procedures exhibit a few undesirable traits. For example, note that if we had switched contracts, the alternative second holding would have been $\widetilde{\omega}_{2}=-\frac{\widetilde{\sigma}_{1,2}}{\widetilde{\sigma}_{2}^{2}}=-\frac{\sigma_{1,2}}{\sigma_{1}^{2}}$, and $\widetilde{\omega}_{2} \neq \frac{1}{\omega_{2}}$ unless $\sigma_{1}=\sigma_{2}$. So the ordering of the instruments introduces some arbitrariness to this solution. Also, the risk contributed by each of the basket's constituents is not equal. In order to evaluate that, we need first to formalize the concept of Contribution to Risk.

\subsection{CONTRIBUTION TO RISK}

The marginal contribution to the basket's variance from a leg $i$ is

$$
\frac{\partial \sigma_{\Delta B}}{\partial \omega_{i}}=\frac{1}{2}\left(\sigma_{\Delta B}^{2}\right)^{-1 / 2} \frac{\partial \sigma_{\Delta B}^{2}}{\partial \omega_{i}}=\frac{\sigma_{\Delta B, \Delta S_{i}}}{\omega_{i} \sigma_{\Delta B}}=\frac{\sigma_{i} \sum_{j=1}^{n} \omega_{j} \sigma_{j} \rho_{i, j}}{\sigma_{\Delta B}}
$$


where $\Delta S_{i}=\Delta P_{i} \omega_{i}$, and $\sigma_{\Delta B, \Delta S_{i}}$ is the covariance between changes in the basket's dollar value and changes in the $i$-th leg's dollar value. Because volatility is a homogeneous function of degree 1 , Euler's theorem allows us to write

$$
\sigma_{\Delta B}=\sum_{i=1}^{n} \omega_{i} \frac{\partial \sigma_{\Delta B}}{\partial \omega_{i}}=\frac{1}{\sigma_{\Delta B}} \sum_{i=1}^{n}[V \omega \circ \omega]_{i}
$$

where $\circ$ represents a Hadamard product. We can define Contribution to Risk $(\mathrm{CtR})$ as

$$
C t R_{i}=\frac{\partial \sigma_{\Delta B}}{\partial \omega_{i}} \frac{\omega_{i}}{\sigma_{\Delta B}}=\frac{[V \omega \circ \omega]_{i}}{\omega^{\prime} V \omega}=\frac{\omega_{i} \sigma_{i} \sum_{j=1}^{n} \omega_{j} \sigma_{j} \rho_{i, j}}{\sigma_{\Delta B}^{2}}=\frac{\sigma_{\Delta B, \Delta S_{i}}}{\sigma_{\Delta B}^{2}}
$$

and obviously $\sum_{i=1}^{n} C t R_{i}=1$. One hedging option would be to estimate the Equal-Risk Contribution (ERC) vector, such that $C t R_{i}=C t R_{j}, \forall i, j$. In our $n=2$ case, that occurs when

$$
\begin{gathered}
h_{1}^{2} \sigma_{1}^{2}+h_{1} \sigma_{1} \omega_{2} \sigma_{2} \rho_{1,2}=\omega_{2}^{2} \sigma_{2}^{2}+\omega_{2} \sigma_{2} h_{1} \sigma_{1} \rho_{1,2} \\
\omega_{2}=-h_{1} \frac{\sigma_{1}}{\sigma_{2}}
\end{gathered}
$$

which leads to $\omega_{E R C} \approx(1000,-1552.48)$. Unlike in the OLS solution, the ERC solution is not affected by the order of the instruments. The risk of ERC's hedging basket is $\sigma_{\Delta B} \approx 303,879.05$. That is very close to the minimum risk of the MV-OLS solution, with the advantage that with ERC we get $C t R_{1}=C t R_{2}=\frac{1}{2}$. That is not the case of $\omega_{M V} \approx(1000,-1467.85)$, for which we obtain $C t R_{1}=1$ and $C t R_{2}=0$. So although the MV-OLS solution has reduced risk from $920,304.74$ to a minimum $299,709.02$, the first leg is still responsible for the entirety of the risk. In order to understand why, we need to introduce the concept of Correlation to Basket (CtB).

\subsection{CORRELATION TO BASKET}

The correlation of each constituent to the overall basket can be computed as

$$
\begin{aligned}
& \sigma_{\Delta S_{i}, \Delta S_{j}}=\omega_{i} \sigma_{i, j} \omega_{j}=\omega_{i} \sigma_{i} \omega_{j} \sigma_{j} \rho_{i, j} \\
& \sigma_{\Delta B, \Delta S_{i}}=\sum_{j=1}^{n} \sigma_{\Delta S_{i}, \Delta S_{j}}=\omega_{i} \sigma_{i} \sum_{j=1}^{n} \omega_{j} \sigma_{j} \rho_{i, j} \\
& C t B_{i}=\rho_{\Delta B, \Delta S_{i}}=\frac{\sigma_{\Delta B, \Delta S_{i}}}{\sigma_{\Delta B} \sigma_{\Delta S_{i}}}=\operatorname{Sgn}\left[\omega_{i}\right] \frac{\sum_{j=1}^{n} \omega_{j} \sigma_{j} \rho_{i, j}}{\sigma_{\Delta B}}=\operatorname{Sgn}\left[\omega_{i}\right] \rho_{\Delta B, i}
\end{aligned}
$$


where $\sigma_{\Delta S_{i}}=\sqrt{\sigma_{\Delta S_{i}}^{2}}=\left|\omega_{i}\right| \sigma_{i}$. The factor $\operatorname{Sgn}\left[\omega_{i}\right]$ arises from simplifying $\frac{\omega_{i}}{\sqrt{\omega_{i}^{2}}}$. López de Prado and Leinweber (2012) show how to compute a general hedging basket such that $C t B_{i}=$ $C t B_{j}, \forall i, j$, which the authors call MMSC (for Mini-Max Subset Correlation). In our particular $n=2$ case, we expect the hedge to be $\omega_{2}<0$ since $\rho_{1,2}>0$, so the equal exposure is achieved at

$$
\begin{gathered}
h_{1} \sigma_{1}+\omega_{2} \sigma_{2} \rho_{1,2}=-\omega_{2} \sigma_{2}-h_{1} \sigma_{1} \rho_{1,2} \\
\omega_{2}=-h_{1} \frac{\sigma_{1}}{\sigma_{2}}
\end{gathered}
$$

which means that the MMSC and ERC solutions coincide when $n=2$. There is a connection between $\mathrm{CtR}$ and $\mathrm{CtB}$, as evidenced by Eq. (6) and Eq. (10), specifically,

$$
C t R_{i}=\frac{\sigma_{\Delta S_{i}}}{\sigma_{\Delta B}} C t B_{i}=\frac{\left|\omega_{i}\right| \sigma_{i}}{\sigma_{\Delta B}} C t B_{i}
$$

From this expression we deduce that an ERC basket will match the MMSC solution when $\left|\omega_{i}\right| \sigma_{i}=\left|\omega_{j}\right| \sigma_{j}$. This condition is clearly met when $n=2$ (see Eq. 11), however that is not generally true when $n>2$.

For $\omega_{M M S C} \approx(1000,-1552.48)$, we obtain $C t B_{1}=C t B_{2} \approx 0.17$, while for $\omega_{M V} \approx$ $(1000,-1467.85)$ we obtained $C t B_{1} \approx 0.33$ and $C t B_{2}=0$. So what the MV-OLS solution has done is to add an "ES1 Index" leg that is orthogonal to the basket. The risk from $\omega_{M V}$ is slightly lower (in fact, the lowest), however it is still concentrated in the first leg $\left(C t R_{1}=1\right)$. These are good reasons for favouring MMSC or ERC solutions over MV or OLS.

\subsection{SPECTRAL DECOMPOSITION}

A change of basis will allow us to understand geometrically the previous analysis. Because a covariance matrix $V$ is squared and symmetric, its eigenvector decomposition delivers a set of real-valued orthonormal vectors that we can use to plot the MV-OLS and the MMSC-ERC solutions. Applying the Spectral Theorem,

$$
V=W \Lambda W^{\prime}
$$

where $\Lambda$ is the eigenvalues matrix, $W$ is the eigenvectors matrix, and $W^{\prime}$ denotes its transpose. $\Lambda$ is a squared diagonal matrix and the columns of $W$ are orthogonal to each other, i.e. $W^{\prime}=W^{-1}$, with unit length. For convenience, we reorder columns in $W$ and $\Lambda$ so that $\Lambda_{i, i} \geq \Lambda_{j, j}, \forall j>i$. The $i$-th principal component is defined by a portfolio with the holdings listed in the $i$-th column of $W$. Looking at the above equation, $\Lambda$ can then be interpreted as the covariance matrix between the principal components characterized by the columns of $W$. The factor loadings vector $f_{\omega}=W^{\prime} \omega$ gives us the projection of $\omega$ into this new orthogonal basis. This can be verified from $\sigma_{\Delta B}^{2}=\omega^{\prime} V \omega=\omega^{\prime} W \Lambda W^{\prime} \omega=f_{\omega}{ }^{\prime} \Lambda f_{\omega}$. The product $f_{I}=W^{\prime} I$, where $I$ represents the identity matrix, gives us the directions of the old axes in the new basis. 
Going back to our original example,

$$
W=\left[\begin{array}{cc}
0.84646 & -0.53245 \\
0.53245 & 0.84646
\end{array}\right], \quad \Lambda=\left[\begin{array}{cc}
1171422.026 & 0 \\
0 & 26946.176
\end{array}\right]
$$

For the initial position $\omega_{0}=\left[\begin{array}{c}1000 \\ 0\end{array}\right]$, the factor loadings are $f_{\omega_{0}} \approx\left[\begin{array}{c}846.46203 \\ -532.44908\end{array}\right]$. It becomes evident that $\omega_{0}$ is not well hedged, because the $f_{\omega_{0}}$ vector is not pointing in the direction of the second orthogonal component, which is the one with least variance. If we adopted the MV-OLS solution, the resulting basket would take the direction $f_{\omega_{M V}} \approx\left[\begin{array}{c}64.90733 \\ -1774.9273\end{array}\right]$ in the orthonormal axes. That is a noticeable improvement over $\omega_{0}$, as $\omega_{M V}$ is shown to be much closer to the second orthogonal component, thus it remains little directional risk in terms of these two instruments ("FA1 Index" and "ES1 Index"). And yet the MMSC-ERC solution is less directional, with $f_{\omega_{M M S C}} \approx\left[\begin{array}{c}19.84512 \\ -1846.56505\end{array}\right]$.

The first component is typically associated with market risk, of which $\omega_{M M S C}$ exhibits the least. The MMSC basket is almost completely associated with spread risk, which is best captured by the second component. López de Prado and Leinweber (2012) show how to compute in general a hedging portfolio with zero exposure to the first components. For the $n=2$ case, it means that

$$
\begin{gathered}
f_{\omega}=W^{\prime}\left[\begin{array}{l}
\omega_{1} \\
\omega_{2}
\end{array}\right]=\left[\begin{array}{c}
0 \\
\frac{\sigma_{\Delta B}}{\sqrt{\Lambda_{2,2}}}
\end{array}\right] \\
{\left[\begin{array}{l}
\omega_{1} \\
\omega_{2}
\end{array}\right]=W\left[\begin{array}{c}
0 \\
\frac{\sigma_{\Delta B}}{\sqrt{\Lambda_{2,2}}}
\end{array}\right]=\left[\begin{array}{l}
W_{1,2} \\
W_{2,2}
\end{array}\right] \frac{\sigma_{\Delta B}}{\sqrt{\Lambda_{2,2}}}}
\end{gathered}
$$

and re-scaling to meet the condition that $\omega_{1}=h_{1}$, we obtain that $\omega_{P C A}=h_{1}\left(1, \frac{W_{2,2}}{W_{1,2}}\right)$. In our example, this means that $\omega_{P C A} \approx(1000,-1589.75)$. Then, $f_{\omega_{P C A}} \approx\left[\begin{array}{c}0 \\ -1878.11386\end{array}\right]$, which indicates that we are perfectly hedged against the most volatile of the orthogonal components (typically known as the "market component"), as expected. Figure 2 graphs these vectors.

\section{[FIGURE 2 HERE]}

The columns of $W$ (which characterize the Principal Components) represent linear combinations of the securities, which happen to be orthogonal to each other, sorted descending in terms of the variance associated with that direction. In this PCA approach, we are hedged against all risk coming from the first $n-1$ components (with the largest variance), and fully concentrated on the $n$-th component (with the smallest variance). One caveat of the PCA approach is that the interpretation of the $n$-th component is not necessarily intuitive in terms of the basket constituents. For example, in the ERC approach we know that each leg contributes equal risk, 
and in the MMSC approach that the basket had equal exposure to each leg. That direct connection between the solution and the basket constituents is missing in the PCA approach, because of the change of basis. This is generally perceived as a drawback, particularly in highlydimensional problems. MMSC is an appealing alternative to PCA because MMSC searches for a basket as orthogonal as possible to the legs, without requiring a basis change (like PCA). So although MMSC's solution is close to PCA's, it can still be linked intuitively to the basket's constituents. Understanding how this is done beyond the two-dimensional framework requires us to introduce the concept of subset correlation.

\section{HEDGING BEYOND TWO DIMENSIONS}

In the previous section we argued in favor of MMSC and ERC over alternative hedging approaches such as MV or PCA. The problem with MV's solution was that risk contribution (measured in terms of CtR) and exposure (measured in terms of CtB) of the basket's constituents is typically unbalanced. The problem with PCA was that the solution cannot be immediately (or for that matter, intuitively) understood in terms of the basket's constituents. In this section we will examine the distinct features of MMSC and ERC beyond the two-dimensional case.

The previous example was simplified by the fact that we were only considering two dimensions. As we will see next, there is a qualitative difference between working in two dimensions and working with three or more. Suppose that a portfolio manager wishes to hedge her position of 1,000 S\&P Midcap 400 E-mini futures contracts ("FA1 Index") using two other instruments: S\&P 500 E-mini futures ("ES1 Index") and DJAI E-Mini futures ("DM1 Index"). The relevant covariance and correlation matrices of daily market value (dollar) changes are shown in Figure 3 , with the corresponding eigenvectors and eigenvalues matrices.

\section{[FIGURE 3 HERE]}

As expected, we can appreciate a high and positive codependence between these three products. It is of course desirable to hedge our position using instruments highly correlated to it (about 0.95 correlation against "ES1 Index", and 0.91 correlation against "DM1 Index"). Unfortunately, that comes at the cost of having to deal with a similarly high correlation between the hedging instruments (about 0.98 correlation between "ES1 Index" and "DM1 Index"). This poses a problem because there may be an overlap between the hedges, which was not present in the twodimensional case.

\subsection{THE EQUAL-RISK CONTRIBUTION (ALIAS, RISK PARITY) SOLUTION}

We will illustrate this last point by computing the ERC basket, which solves the problem (recall Eq. (6))

$$
\omega_{E R C}=\left\{\omega_{i} \mid \frac{\sigma_{\Delta B, \Delta S_{i}}}{\sigma_{\Delta B}^{2}}=\frac{1}{n}, \forall i\right\}
$$

Appendix 1 provides the details of this calculation, for any $n$ dimensions, and Appendix 2 offers an algorithm coded in Python which computes the ERC basket. 


\section{[FIGURE 4 HERE]}

Figure 4 reports the results of applying this algorithm to the input variables in Figure 3 . A first problem with this result is the uneven correlations to the basket (CtB). The ("ES1 Index", "DM1 Index") subset will dominate the performance of the hedge, with its 0.26 correlation to the basket. This could have potentially serious consequences should there exist a correlation break between "ES1 Index" and "DM1 Index" on one hand and "FA1 Index" on the other. A second problem is that the solution itself is not unique. Figure 5 presents an alternative solution for which also $C t R_{i} \approx \frac{1}{3}, \forall i$, with unacceptably high values like $C t B_{2}>0.99$. We would of course reject this alternative solution out of common sense, however it would be better to rely on a procedure that searches for reasonable hedges, if possible with unique solutions.

\section{[FIGURE 5 HERE]}

In conclusion, ERC does not necessarily deliver a unique and balanced (exposure-wise) solution when $n>2$.

\subsection{DIVERSIFIED RISK PARITY}

Building on an idea of Meucci (2009b), Lohre, Neugebauer and Zimmer (2012) and Lohre, Opfer, and Ország (2012), proposed a very interesting variation of ERC that they branded "Diversified Risk Parity" (DRP). It computes the allocations such that the contribution to risk from every principal component is equal. In a nutshell, DRP is like ERC, but computed on the principal components instead of the actual instruments. Like PCA, DRP also requires a spectral decomposition, and thus a change of basis. We cannot strictly classify it as a balanced basket, however we will derive its calculation for illustration purposes. Our DRP solution differs from Lohre, Neugebauer and Zimmer (2012) in two aspects: First, we are not imposing the asset allocation constraints (non-negativity, additivity to one), because they are not relevant in a hedging framework. Second (and as a consequence of the first aspect), our solution is analytical, while theirs is numerical.

\subsubsection{ANALYTICAL SOLUTION}

Recall from Section 2.3 that the eigenvectors of a symmetric matrix are orthonormal, thus $W^{\prime} W=I$ and $\Lambda_{i, j}=0, \forall i \neq j$. The correlation between any two distinct principal components is $\frac{\Lambda_{i, j}}{\sqrt{\Lambda_{i, i} \Lambda_{j, j}}}=0, \forall i \neq j$. This implies that, given some factor loadings $f_{\omega}=W^{\prime} \omega$, the basket's variance can be simply decomposed as $\sigma_{\Delta B}^{2}=\sum_{i=1}^{n}\left[f_{\omega}\right]_{i}^{2} \Lambda_{i, i}$, where $\left[f_{\omega}\right]_{i}$ is the $i$-th element of the factor loading's vector. We conclude that the contribution to risk of the $i$-th principal component is

$$
\widetilde{C t} R_{i}=\frac{\left[f_{\omega}\right]_{i}^{2} \Lambda_{i, i}}{\sum_{j=1}^{n}\left[f_{\omega}\right]_{j}^{2} \Lambda_{j, j}}
$$

Suppose that $\left[f_{\omega}\right]_{i}=\sqrt{\frac{\Lambda_{1,1}}{\Lambda_{i, i}}}, \forall i$. Then, $\widetilde{C t R_{i}}=\frac{\frac{\Lambda_{1,1}}{\Lambda_{i, i}} \Lambda_{i, i}}{\Lambda_{1,1} \sum_{j=1}^{n} \frac{1}{\Lambda_{j, j}} \Lambda_{j, j}}=\frac{1}{n}$, which is the ERC solution on factor loadings $f_{\omega}$. But $f_{\omega}$ are loadings in the new basis (of principal components), and we still 
need to derive the holdings in the old basis (of actual instruments). Since $f_{\omega}=W^{\prime} \omega$ and $W^{\prime}=W^{-1}$, these can be computed as

$$
\omega_{D R P}=W^{\prime-1} f_{\omega}=W f_{\omega}
$$

\section{[FIGURE 6 HERE]}

In our particular example, Eq. (18) leads to the solution shown in Figure 6. This result is mathematically correct, and yet looking at the actual holdings proposed, many portfolio managers may have a difficult problem understanding it. $\widetilde{C t} R_{i}=\frac{1}{3}, \forall i$, however $\mathrm{CtR}$ and $\mathrm{CtB}$ (in terms of the instruments) exhibit a large concentration on "DM1 Index". Another inconvenience of DRP, which in fact is shared by all PCA-style approaches, is their lack of robustness in the presence of numerically ill-conditioned covariance matrices: A change in just one observation may produce a last eigenvector that spans in a completely different direction, and because DRP equally distributes risk among all principal components, the impact on $\omega_{D R P}$ will be dramatic. This makes PCA-like baskets in general, and DRP in particular, vulnerable to structural breaks and outliers. In Appendix 5 we present a covariance clustering procedure which addresses this concern.

\subsubsection{THE DANGER OF "HOLDING" AN EIGENVECTOR IN A RISK-ON/RISK-OFF ENVIRONMENT}

Regardless of the practicality of the DRP solution, it is useful for understanding why baskets pointing in the direction of an eigenvector should not be part of a hedging basket, except for the eigenvector associated with the smallest eigenvalue. Denote by $\sigma\left[W_{i}\right]$ the risk of a portfolio pointing in the direction of the $i$-th eigenvector, and by $\sigma\left[\frac{\omega_{D R P}}{\left\|\omega_{D R P}\right\|}\right]$ the risk associated with a unitlength vector pointing in the direction of the DRP solution. As we move away from $\frac{\omega_{D R P}}{\left\|\omega_{D R P}\right\|}$ and toward $W_{i}$, we have computed the intermediate baskets, $\omega_{\alpha}=\alpha W_{i}+(1-\alpha) \frac{\omega_{D R P}}{\left\|\omega_{D R P}\right\|}$, which can be normalized as $u_{\alpha}=\frac{\omega_{\alpha}}{\left\|\omega_{\alpha}\right\|}$. Figure 7(a) plots the resulting risk, $\sigma\left[u_{\alpha}\right]$ for $\alpha \in[0,1]$ in 100 equally spaced nodes. Risk increases as we approach $W_{1}$ and $W_{2}$, but not $W_{3}$. This is because eigenvectors are the critical points of the Rayleigh quotient $\frac{\omega^{\prime} V \omega}{\omega^{\prime} \omega}$, where the numerator is the variance of the basket. Consider the optimization program $\max _{\omega} \omega^{\prime} V \omega$, subject to $\omega^{\prime} \omega=1$, with a Lagrangian $\mathcal{L}(\omega, \lambda)=\omega^{\prime} V \omega-\lambda\left(\omega^{\prime} \omega-1\right)$. Applying first order conditions on $\omega$, $\frac{\partial \mathcal{L}(\omega, \lambda)}{\omega}=\left(V+V^{\prime}\right) \omega-2 \lambda \omega=2 V \omega-2 \lambda \omega=0 \Rightarrow V \omega=\lambda \omega$. But that is precisely the generalized eigenvalue problem. So, finding the largest eigenvalue of $V, \lambda=\Lambda_{1,1}=W_{1}^{\prime} V W_{1}$, leads us to the maximum, which is achieved by the first eigenvector, $W_{1}$. Furthermore, because $V W=W \Lambda \Rightarrow \Lambda_{i, i}=\frac{W_{i}^{\prime} V W_{i}}{W_{i}^{\prime} W_{i}}$, gives a Rayleigh quotient, all critical points (and extreme values in particular) of this optimization program are derived from computing the eigenvectors of $V$, with stationary values in $\Lambda$.

[FIGURE 7(a) HERE] 
Because $V W=W \Lambda$, the eigenvector makes the covariance matrix behave like a mere scalar. A portfolio that concentrates risk in the direction of a particular eigenvector $W_{i}$ (except for $i=n$ ), is investing in a single bet. This means that the investment universe that forms $V$ will not be able to dissipate a "hit" that comes from the direction of $W_{i}$, a situation particularly dangerous in a riskon/risk-off environment. Figure 7(b) displays the risk of a portfolio that moves away from DRP and toward $W_{1}$, before (risk-off) and after (risk-on) a $100 \%$ increase in $\sqrt{\Lambda_{1,1}}$ (standard deviation in the direction of $W_{1}$ ). As we can see, investors holding the first eigenvector receive the entirety of the shock, and their risk is doubled. That shock would have been greatly dissipated by the investment universe if the investor had held a portfolio closer to DRP, instead of being so exposed to the first eigenvector.

\section{[FIGURE 7(b) HERE]}

\subsection{THE MAXIMUM-DIVERSIFICATION SOLUTION}

Choueifaty and Coignard (2008) compute the vector of holdings that Maximize the Diversification Ratio (MDR), as defined by

$$
\omega_{M D R}=\arg \max _{\omega} \frac{\sum_{i=1}^{n} \omega_{i} \sigma_{i}}{\sigma_{\Delta B}}
$$

This diversification ratio is the ratio of weighted volatilities divided by the basket's volatility, and it is closely related to our Eq. (10). MDR is an intuitive method that penalizes the risk associated with cross-correlations, as they are accounted by the denominator but absent in the numerator of the maximized ratio. Choueifaty, Froidure and Reynier (2011) show that the correlations of each leg to the MDR hedging basket are minimized and made equal. ${ }^{1}$ Figure 8 presents the MDR solution to our example. $C t B_{i} \approx 0.08, \forall i$, and there is no way we can make $\mathrm{CtB}$ smaller for all legs. Now $\mathrm{CtR}$ values make more sense, because the leg with a negative holding is responsible for almost $1 / 2$ of the total risk, with the other half going to the legs with a positive holding. Among the "long" legs the risk is not equally spread, because the correlation between "DM1 Index" and "ES1 Index" (about 0.98) is greater than the correlation between "FA1 Index" and "ES1 Index" (about 0.95). So the MDR result seems intuitive and preferable to the ERC result.

\section{[FIGURE 8 HERE]}

However, if we look more closely into this MDR result, we will find something not entirely satisfactory. The problem is, there are subsets of instruments that, combined, exhibit greater correlation to the overall basket. Figure 8 shows that, even though the exposure is perfectly balanced at the leg level, this hedging basket's performance may still be dominated by some groups of legs. In particular, there is an approx. 0.36 correlation between the subset made of ("ES1 Index", "DM1 Index") and the overall basket. Like in the ERC case, that could be a source of losses should there be a correlation break between large-cap and mid-cap stocks, as witnessed most recently during the 2008 financial crisis. Furthermore, Choueifaty and Coignard (2008)

\footnotetext{
${ }^{1}$ As we will show in the next Section, this is equivalent to a MMSC where only subsets of one instrument are taken into consideration.
} 
acknowledge that the solution may not be unique or robust, particularly with ill-conditioned covariance matrices. Adding some structure to the optimization program would alleviate these problems.

Succinctly, although MDR is to some extent preferable to ERC, it does not address the problems of uniqueness of solution and balanced exposure of subsets of legs to the overall basket.

\subsection{THE MINI-MAX SUBSET CORRELATION SOLUTION}

We denote subset correlation as the correlation of a subset of instruments to the overall basket. MMSC's goal is to prevent that any leg or subset of legs dominates the basket's performance, as measured by its subset correlations. This additional structure adds the robustness and uniqueness of solution that were missing in ERC and MDR. MMSC baskets are also more resilient to structural breaks, because this approach minimizes the basket's dependency to any particular leg or subset of legs. Suppose for instance that $\Lambda_{3,3}$ rises and as a result the correlation of the basket to those legs and subsets most exposed to the third principal component increases by a function of $\Delta \Lambda_{3,3}$. Because MMSC provided the most balanced exposure, it will generally be the least impacted basket. We will illustrate this point with an example in Section 3.5.

When we were dealing with 2 instruments, the only subsets were the instruments themselves, so the only subset correlations were the CtBs. The MMSC solution coincided with the MDR solution. But now that $n=3$, we can compute correlations to 6 subsets (the 3 single legs plus the 3 possible pairs of legs), and we need to distinguish between both procedures. The solution can be characterized as

$$
\omega_{M M S C}=\arg \min _{\omega}\left\{\max _{i}\left|\rho_{\Delta B, \Delta S_{i}}\right|\right\}
$$

where $i=1, \ldots, N$ subsets, $N=\sum_{i=1}^{n-1}\left(\begin{array}{l}n \\ i\end{array}\right)=2\left(2^{n-1}-1\right)$ is the number of subsets (excluding the empty set and the full set), $\Delta S_{i}=\sum_{j=1}^{n} \Delta P_{j} \breve{\omega}_{i, j}$, and $\breve{\omega}_{i}$ is the vector of holdings of subset $i$. For example, if the $i$-th subset is formed by instruments 1 and $2, \widetilde{\omega}_{i}$ will be a vector with entries $\breve{\omega}_{i, 1}=\omega_{1}, \breve{\omega}_{i, 2}=\omega_{2}$ and $\breve{\omega}_{i, j}=0$ for $2<j \leq n$.

$N>n$ when $n>2$. As $n$ grows, we will have many more subsets $(N)$ than instruments $(n)$. Ideally we would like to minimize all subset correlations and bring them as close to each other as possible, hence the name MMSC (Mini-Max Subset Correlations). Appendix 3 presents an algorithm for computing the MMSC solution for any dimension, and Appendix 4 provides the code in Python. Figure 9 shows the results for our example. The greatest correlation of any subset to the overall basket is about 0.185, significantly lower than in the ERC and MDR cases.

\section{[FIGURE 9 HERE]}

$C t R_{1} \approx \frac{1}{2}$ seems to point at a concentration of risk in the "FA1 Index" leg. This is an artifact of computing $\mathrm{CtR}$ when one of the legs has low correlation to the basket. In this instance, CtR will not be able to accurately split risk among the instruments. From Appendix 1 we know that 


$$
\frac{\partial C t R_{j}}{\partial \omega_{j}}=\frac{\omega_{j} \sigma_{j}^{2}}{\sigma_{\Delta B}^{2}}\left(1-2 \rho_{\Delta B, \Delta S_{j}}^{2}\right)+\frac{\sigma_{j} \rho_{\Delta B, \Delta S_{j}}}{\sigma_{\Delta B}}
$$

$\frac{\partial C t R_{2}}{\partial \omega_{2}} \approx \frac{\omega_{2} \sigma_{2}^{2}}{\sigma_{\Delta B}^{2}}$ because $\rho_{\Delta B, \Delta S_{2}}^{2} \approx 0 . \frac{\sigma_{j}}{\sigma_{\Delta B}}>1$, because this is a hedging basket. Thus, a very small change in the holdings could transfer a substantial amount from $C t R_{1}$ to $C t R_{2}$, which is counterintuitive. We can also see that, despite the high $C t R_{1}$ value, $C t B_{1}$ is virtually the same as the correlation of the subset formed by ("FA1 Index", "ES1 Index") to the basket, or the correlation of the subset ("ES1 Index", "DM1 Index") to the basket. In contrast, CtB is more stable, with $\frac{\partial \rho_{\Delta B, \Delta S_{2}}}{\partial \omega_{2}} \approx \frac{\sigma_{2}}{\sigma_{\Delta B}}$. The derivative of the correlation to the basket does not have a factor $\omega_{j}$ or a second power on $\frac{\sigma_{j}}{\sigma_{\Delta B}}>1$, thus it is more resilient to small changes in $\omega_{2}$ (another reason why MMSC will tend to be more robust than ERC): ${ }^{2}$

$$
\frac{\partial \rho_{\Delta B, \Delta S_{j}}}{\partial \omega_{j}}=\frac{\sigma_{j}}{\sigma_{\Delta B}}\left(1-\rho_{\Delta B, \Delta S_{j}}^{2}\right)
$$

Summarily, MMSC provides the basket with the most balanced exposure to any of its legs or subsets of legs. The solution is also unique and more robust, virtue of the 6 conditions imposed on only 2 holdings. The fact that $N \gg n$ as $n$ grows provides a competitive advantage for users with access to high performance computing (HPC) facilities, who can deploy a solution unavailable to investors with limited computational power. When $n>30$, the computations involved would present a challenge for today's supercomputers. However, that obstacle could be surmounted by clustering the legs into highly correlated blocks, as proposed in Appendix 5. A second alternative is to limit the maximum size of the subsets evaluated, which can be done through the parameter maxSubsetSize in the Python code provided in Appendix 4.

\subsection{GEOMETRIC INTERPRETATION}

As we did in the two-dimensional case, we can understand the previous three-dimensional results in geometric terms. For the initial position $\omega_{0}=\left[\begin{array}{c}1000 \\ 0 \\ 0\end{array}\right]$, the factor loadings are $f_{\omega_{0}} \approx$ $\left[\begin{array}{c}774.88384 \\ -620.81023 \\ 118.95250\end{array}\right]$. As expected, $\omega_{0}$ is not well-hedged, because the $f_{\omega_{0}}$ vector is nowhere close to the orthogonal directions with least variance. ERC is better hedged, with $f_{\omega_{E R C}} \approx$ $\left[\begin{array}{c}26.23808 \\ -558.67818 \\ 5320.06831\end{array}\right]$. For MDR, we get $f_{\omega_{M D R}} \approx\left[\begin{array}{c}14.14535 \\ -388.45011 \\ 6287.25928\end{array}\right]$, and for MMSC we get $f_{\omega_{M M S C}} \approx$ $\left[\begin{array}{c}33.59253 \\ -691.79627 \\ 4577.41954\end{array}\right]$. So it would appear as if, in this particular example, MMSC did a worse job than MDR and ERC. Figure 10 tells us that this is not the case.

\footnotetext{
${ }^{2}$ See López de Prado and Leinweber (2012) for a proof.
} 


\section{[FIGURE 10 HERE]}

The first table reports the factor loadings of the three balanced hedging baskets to the first principal component. Although $f_{\omega_{M M S C}}(1) \approx 33.59253$ is slightly greater than for the other baskets, when it comes to the subsets we find that $f_{\omega_{M M S C}}$ is generally the least exposed. This is an important feature because, in the case of a structural break ${ }^{3}, \mathrm{MMSC}^{\prime}$ 's hedging basket will tend to be the least impacted. Figure 10 shows that MMSC is the least exposed to shocks in the direction of any principal component. This is the result of minimizing the correlation of any subset to the overall basket. From Figures 4, 8 and 9, we know that all three balanced baskets had the greatest exposure to subset $(2,3)$. This subset, composed of ("ES1 Index", "DM1 Index"), is particularly sensitive to shocks in the direction of the third principal component (see panel 3 in Figure 10). Figure 11 displays the covariance and correlation matrices that result from a shock in the direction of that third principal component, in particular a $25 \%$ increase to $\Lambda_{3,3}$. The differences between both correlation matrices seem negligible, and yet the impact on ERC and MDR is significant. Figure 12 reports how, as a consequence of this structural break, the correlations of each balanced basket to subset $(2,3)$ are impacted. As expected, MMSC is the only basket with a relatively low exposure of around 0.23 (compared to the previous 0.19 ), while ERC and MDR's go up to approx. 0.31 and 0.41 respectively.

\section{[FIGURE 11 HERE]}

\section{[FIGURE 12 HERE]}

\section{TRADING BASKETS}

As we have seen earlier, a hedging basket attempts to minimize the exposure to any of its constituents. In contrast, a trading basket tries to determine the holdings such that the exposure is maximized. With that difference in mind, the problem is again how to determine a basket with balanced exposures, i.e. that no particular leg or subset of legs is responsible for the overall basket's performance.

As discussed earlier, MDR's goal is to maximize diversification by minimizing all CtB. This is consistent with a hedging problem. As originally formulated, it cannot be used to compute a trading basket.

The ERC procedure can sometimes deliver a trading basket by chance. The result reported in Figure 5 happened to be a trading basket, even though we were searching for a hedging basket. This is because ERC does not have a way to control for those two different objectives. ERC may converge to one or the other depending on the initial seed (e.g., a vector of ones in the case of Figure 5). This problem could be circumvented by trying different seeds. As expected, the resulting ERC trading basket for our example is balanced in terms of contribution to risk (CtR).

\footnotetext{
${ }^{3}$ In this context, we speak of a "structural break" with the meaning of a change in the direction of a principal component. This is what occurs if, for example, we stretch the location-dispersion ellipsoid in the direction of an eigenvector. See Meucci (2009a), Chapter 3.
} 
When used to compute a trading basket, MMSC will maximize the minimum subset correlation (as opposed to minimizing the maximum subset correlation). In every iteration, it will push the lowest subset correlation to a higher value, thus raising the average correlation, until there is no way to increase any correlation without reducing another.

$$
\omega=\arg \max _{\omega}\left\{\min _{i}\left|\rho_{\Delta B, \Delta S_{i}}\right|\right\}
$$

where $i=1, \ldots, N$ subsets, $N=\sum_{i=1}^{n-1}\left(\begin{array}{c}n \\ i\end{array}\right)=2\left(2^{n-1}-1\right)$ is the number of subsets, $\Delta S_{i}=$ $\sum_{j=1}^{n} \Delta P_{j} \breve{\omega}_{i, j}$, and $\breve{\omega}_{i}$ is the vector of holdings of subset $i$. Figure 13 shows the trading basket computed by MMSC on the same three instruments used in the previous section. MMSC recognizes that a linear combination of "FA1 Index" and "DM1 Index" makes "ES1 Index" redundant, thus giving $\omega_{2} \approx 0$. As a result, MMSC spreads risk equally between "FA1 Index" and "DM1 Index", rather than between three instruments (including a redundant position on "ES1 Index"), like the ERC approach did. Perhaps more important is that MMSC actively searches for this trading basket, rather than arriving at it thanks to a fortunate seed. Appendix 4 provides the Python code that computes MMSC hedging and trading baskets, depending on the user's preference.

[FIGURE 13 HERE]

\section{CONCLUSIONS}

PCA is a good theoretical option for computing hedging baskets. One drawback of the PCA approach is that the interpretation of the $n$-th component is not necessarily intuitive in terms of the basket's constituents. This is due to the change of basis involved in PCA's eigendecomposition of variance, and for $n>3$ it is usually difficult to visualize the intuition behind the resulting eigenvectors. A case in point was the solution delivered by the DRP basket which, although mathematically correct, it defied common sense.

For this reason, practitioners typically rely on approaches that allow them to relate the basket's holdings to the statistical properties of the basket's constituents. Such is the purpose of balanced hedging baskets, which are characterized by spreading risk or exposure across the baskets' constituents, so that the combined risk is not only minimal but also well distributed. Although the solution is not a perfect hedge (i.e., a minimum variance portfolio orthogonal to the main principal components), there is no change of basis involved and therefore the basket can be understood in terms of its constituents.

How well risk is spread is measured by the Contribution to Risk (CtR) from each leg. Similarly, how well exposure is spread is measured by each leg's Correlation to the Basket (CtB). Three methods have been proposed to compute balanced hedging baskets: Equal-Risk Contribution (ERC), Maximum Diversification (MDR) and Mini-Max Subset Correlation (MMSC). All three are theoretically sound and useful procedures, and the purpose of this paper is not to disqualify any of them but to examine the properties of their respective solutions. 
ERC computes the vector of holdings such that the CtR of each leg is equal. Absent of constraints, the solution is not unique and it could lead to hedging baskets riskier than any of the individual holdings. This problem can be circumvented by trying alternative seeds, however the procedure per se does not control the outcome. Also, although the solution always delivers equal $\mathrm{CtR}$ per leg, the $\mathrm{CtB}$ could be very high for some legs, evidencing concentration of exposure.

MDR is equivalent to ERC, with the difference that instead of equalizing $\mathrm{CtR}$, it attempts to achieve equal $\mathrm{CtB}$ per leg. Solutions tend to be more intuitive and robust, however they are not unique. Another caveat is that, although each leg's CtB may be equal, there may be subsets of legs that are highly exposed, i.e. they will dominate the overall basket's performance.

MMSC computes a hedging basket for which not only $\mathrm{CtB}$ are as low as possible, but also the correlations of subsets of legs to the overall basket are minimized. The outcome is a basket which performance is not dominated by any of its constituents, individually or in subsets. This feature is important, because it makes the basket more resilient to structural breaks. Since the number of subsets of legs is necessarily greater than the number of legs, the system is overdetermined: There may be no solution that equalizes all subset correlations, in which case MMSC computes the Mini-Max approximation. Another advantage of MMSC is that it can also be used to compute trading baskets. These are characterized by a vector of holdings such that the correlation of each leg or subset of legs to the overall basket is maximized (rather than minimized, like in the hedging case).

One caveat of MMSC is that, for very large baskets, the number of subsets can be enormous, and the calculation of its solution may require access to high performance computing (HPC) facilities, such as the NERSC facility at Lawrence Berkeley National Laboratory. ${ }^{4}$ We hope that readers will find helpful the numerically efficient algorithms provided in the Appendices.

\footnotetext{
${ }^{4}$ Additional details are available at: http://www.nersc.gov/users/computational-systems/
} 


\section{APPENDICES}

\section{A.1. COMPUTING THE ERC BASKET FOR $\boldsymbol{n}$-INSTRUMENTS}

\section{A.1.1. TAYLOR'S EXPANSION}

A second-degree Taylor expansion of the CtR function takes the form:

$$
\Delta C t R_{i}=\frac{\partial C t R_{i}}{\partial \omega_{i}} \Delta \omega_{i}+\frac{1}{2} \frac{\partial^{2} C t R_{i}}{\partial \omega_{i}^{2}}\left(\Delta \omega_{i}\right)^{2}+\sum_{k=3}^{\infty} \frac{1}{k !} \frac{\partial^{k} C t R_{i}}{\partial \omega_{i}^{k}}\left(\Delta \omega_{i}\right)^{k}
$$

We therefore need to compute an analytical expression for the first and second partial derivatives.

\section{A.1.2. FIRST DERIVATIVE}

From Eq. (6) we know that $C t R_{i}=\frac{\partial \sigma_{\Delta B}}{\partial \omega_{i}} \frac{\omega_{i}}{\sigma_{\Delta B}}$, thus

$$
\frac{\partial C t R_{i}}{\partial \omega_{i}}=\frac{\partial\left[\frac{\partial \sigma_{\Delta B}}{\partial \omega_{i}} \frac{\omega_{i}}{\sigma_{\Delta B}}\right]}{\partial \omega_{i}}=\frac{\partial^{2} \sigma_{\Delta B}}{\partial \omega_{i}^{2}} \frac{\omega_{i}}{\sigma_{\Delta B}}+\frac{\partial\left[\frac{\omega_{i}}{\sigma_{\Delta B}}\right]}{\partial \omega_{i}} \frac{\partial \sigma_{\Delta B}}{\partial \omega_{i}}
$$

We already know that $\frac{\partial \sigma_{\Delta B}}{\partial \omega_{i}}=\frac{\sigma_{\Delta B, \Delta S_{i}}}{\omega_{i} \sigma_{\Delta B}}$ from Eq. (4). We need to compute $\frac{\partial^{2} \sigma_{\Delta B}}{\partial \omega_{i}{ }^{2}}$ and $\frac{\partial\left[\frac{\omega_{i}}{\sigma_{\Delta B}}\right]}{\partial \omega_{i}}$. From López de Prado and Leinweber (2012) we also know that $\frac{\partial \sigma_{\Delta B}}{\partial \omega_{i}}=\sigma_{i} \rho_{\Delta B, \Delta S_{i}}$ and $\frac{\partial \rho_{\Delta B, \Delta S_{i}}}{\partial \omega_{i}}=$ $\frac{\sigma_{i}}{\sigma_{\Delta B}}\left(1-\rho_{\Delta B, \Delta S_{i}}^{2}\right)$, thus

$$
\begin{gathered}
\frac{\partial^{2} \sigma_{\Delta B}}{\partial \omega_{i}{ }^{2}}=\frac{\partial \rho_{\Delta B, \Delta S_{i}}}{\partial \omega_{i}} \sigma_{i}=\frac{\sigma_{i}^{2}}{\sigma_{\Delta B}}\left(1-\rho_{\Delta B, \Delta S_{i}}^{2}\right) \\
\frac{\partial\left[\frac{\omega_{i}}{\sigma_{\Delta B}}\right]}{\partial \omega_{i}}=\frac{\sigma_{\Delta B}-\frac{\partial \sigma_{\Delta B}}{\partial \omega_{i}} \omega_{i}}{\sigma_{\Delta B}^{2}}=\frac{1}{\sigma_{\Delta B}}-\frac{\omega_{i} \sigma_{i} \rho_{\Delta B, \Delta S_{i}}}{\sigma_{\Delta B}^{2}}
\end{gathered}
$$

We therefore conclude that

$$
\begin{gathered}
\frac{\partial C t R_{i}}{\partial \omega_{i}}=\frac{\omega_{i} \sigma_{i}^{2}}{\sigma_{\Delta B}^{2}}\left(1-\rho_{\Delta B, \Delta S_{i}}^{2}\right)+\left(\frac{1}{\sigma_{\Delta B}}-\frac{\omega_{i} \sigma_{i} \rho_{\Delta B, \Delta S_{i}}}{\sigma_{\Delta B}^{2}}\right) \sigma_{i} \rho_{\Delta B, \Delta S_{i}} \\
=\frac{\omega_{i} \sigma_{i}^{2}}{\sigma_{\Delta B}^{2}}\left(1-2 \rho_{\Delta B, \Delta S_{i}}^{2}\right)+\frac{\sigma_{i} \rho_{\Delta B, \Delta S_{i}}}{\sigma_{\Delta B}}
\end{gathered}
$$




\section{A.1.3. SECOND DERIVATIVE}

Next, we will obtain the expression for

$$
\frac{\partial^{2} C t R_{i}}{\partial \omega_{i}^{2}}=\frac{\partial\left[\frac{\omega_{i}}{\sigma_{\Delta B}^{2}}\right]}{\partial \omega_{i}} \sigma_{i}^{2}\left(1-2 \rho_{\Delta B, \Delta S_{i}}^{2}\right)-4 \rho_{\Delta B, \Delta S_{i}} \frac{\partial \rho_{\Delta B, \Delta S_{i}}}{\partial \omega_{i}} \frac{\omega_{i} \sigma_{i}^{2}}{\sigma_{\Delta B}^{2}}+\frac{\partial\left[\frac{\rho_{\Delta B, \Delta S_{i}}}{\sigma_{\Delta B}}\right]}{\partial \omega_{i}} \sigma_{i}
$$

We need to determine the analytical expressions for $\frac{\partial\left[\frac{\omega_{i}}{\sigma_{\Delta B}^{2}}\right]}{\partial \omega_{i}}$ and $\frac{\partial\left[\frac{\rho_{\Delta B, \Delta S_{i}}}{\sigma_{\Delta B}}\right]}{\partial \omega_{i}}$ :

$$
\begin{gathered}
\frac{\partial\left[\frac{\omega_{i}}{\sigma_{\Delta B}^{2}}\right]}{\partial \omega_{i}}=\frac{1}{\sigma_{\Delta B}^{2}}-2 \frac{\omega_{i} \sigma_{i} \rho_{\Delta B, \Delta S_{i}}}{\sigma_{\Delta B}^{3}} \\
\frac{\partial\left[\frac{\rho_{\Delta B, \Delta S_{i}}}{\sigma_{\Delta B}}\right]}{\partial \omega_{i}}=\frac{\frac{\partial \rho_{\Delta B, \Delta S_{i}}}{\partial \omega_{i}} \sigma_{\Delta B}-\frac{\partial \sigma_{\Delta B}}{\partial \omega_{i}} \rho_{\Delta B, \Delta S_{i}}}{\sigma_{\Delta B}^{2}}=\frac{\sigma_{i}}{\sigma_{\Delta B}^{2}}\left(1-2 \rho_{\Delta B, \Delta S_{i}}^{2}\right)
\end{gathered}
$$

with the conclusion that

$$
\begin{aligned}
\frac{\partial^{2} C t R_{i}}{\partial \omega_{i}^{2}}=\left(\frac{1}{\sigma_{\Delta B}^{2}}\right. & \left.-2 \frac{\omega_{i} \sigma_{i} \rho_{\Delta B, \Delta S_{i}}}{\sigma_{\Delta B}^{3}}\right) \sigma_{i}^{2}\left(1-2 \rho_{\Delta B, \Delta S_{i}}^{2}\right) \\
& -4 \rho_{\Delta B, \Delta S_{i}} \frac{\sigma_{i}}{\sigma_{\Delta B}}\left(1-\rho_{\Delta B, \Delta S_{i}}^{2}\right) \frac{\omega_{i} \sigma_{i}^{2}}{\sigma_{\Delta B}^{2}}+\frac{\sigma_{i}}{\sigma_{\Delta B}^{2}}\left(1-2 \rho_{\Delta B, \Delta S_{i}}^{2}\right) \sigma_{i} \\
& =\frac{\sigma_{i}^{2}}{\sigma_{\Delta B}^{2}}-\frac{\sigma_{i}^{2}}{\sigma_{\Delta B}^{2}} 2 \rho_{\Delta B, \Delta S_{i}}^{2}-2 \frac{\omega_{i} \sigma_{i}^{3} \rho_{\Delta B, \Delta S_{i}}}{\sigma_{\Delta B}^{3}}+4 \frac{\omega_{i} \sigma_{i}^{3} \rho_{\Delta B, \Delta S_{i}}^{3}}{\sigma_{\Delta B}^{3}} \\
& -4 \rho_{\Delta B, \Delta S_{i}} \frac{\omega_{i} \sigma_{i}^{3}}{\sigma_{\Delta B}^{3}}+4 \rho_{\Delta B, \Delta S_{i}}^{3} \frac{\omega_{i} \sigma_{i}^{3}}{\sigma_{\Delta B}^{3}}+\frac{\sigma_{i}^{2}}{\sigma_{\Delta B}^{2}}-\frac{\sigma_{i}^{2}}{\sigma_{\Delta B}^{2}} 2 \rho_{\Delta B, \Delta S_{i}}^{2} \\
= & 2 \frac{\sigma_{i}^{2}}{\sigma_{\Delta B}^{2}}\left(1-2 \rho_{\Delta B, \Delta S_{i}}^{2}\right)-2 \rho_{\Delta B, \Delta S_{i}} \frac{\omega_{i} \sigma_{i}^{3}}{\sigma_{\Delta B}^{3}}\left(2-3 \rho_{\Delta B, \Delta S_{i}}^{2}\right)
\end{aligned}
$$

\section{A.1.4. STEP SIZE}

Finally, assuming $\sum_{k=3}^{\infty} \frac{1}{k !} \frac{\partial^{k} C t R_{i}}{\partial \omega_{i}{ }^{k}}\left(\Delta \omega_{i}\right)^{k} \approx 0$, we can replace these derivatives into Taylor's expansion:

$$
\Delta C t R_{i}=\frac{\partial C t R_{i}}{\partial \omega_{i}} \Delta \omega_{i}+\frac{1}{2} \frac{\partial^{2} C t R_{i}}{\partial \omega_{i}^{2}}\left(\Delta \omega_{i}\right)^{2}
$$

giving us the expression 


$$
\begin{aligned}
\Delta C t R_{i}=\left[\frac{\omega_{i} \sigma_{i}^{2}}{\sigma_{\Delta B}^{2}}\left(1-2 \rho_{\Delta B, \Delta S_{i}}^{2}\right)+\frac{\sigma_{i} \rho_{\Delta B, \Delta S_{i}}}{\sigma_{\Delta B}}\right] \Delta \omega_{i} \\
\quad+\left[\frac{\sigma_{i}^{2}}{\sigma_{\Delta B}^{2}}\left(1-2 \rho_{\Delta B, \Delta S_{i}}^{2}\right)-\rho_{\Delta B, \Delta S_{i}} \frac{\omega_{i} \sigma_{i}^{3}}{\sigma_{\Delta B}^{3}}\left(2-3 \rho_{\Delta B, \Delta S_{i}}^{2}\right)\right]\left(\Delta \omega_{i}\right)^{2}
\end{aligned}
$$

Let us define

$$
\begin{gathered}
a=\frac{\sigma_{i}^{2}}{\sigma_{\Delta B}^{2}}\left(1-2 \rho_{\Delta B, \Delta S_{i}}^{2}\right)-\rho_{\Delta B, \Delta S_{i}} \frac{\omega_{i} \sigma_{i}^{3}}{\sigma_{\Delta B}^{3}}\left(2-3 \rho_{\Delta B, \Delta S_{i}}^{2}\right) \\
b=\frac{\omega_{i} \sigma_{i}^{2}}{\sigma_{\Delta B}^{2}}\left(1-2 \rho_{\Delta B, \Delta S_{i}}^{2}\right)+\frac{\sigma_{i} \rho_{\Delta B, \Delta S_{i}}}{\sigma_{\Delta B}} \\
c=-\Delta C t R_{i}
\end{gathered}
$$

Then, for $a \neq 0$ we will choose the smallest step size (to reduce the error due to Taylor's approximation, which grows with $\left.\left|\Delta \omega_{i}\right|\right)$ :

$$
\Delta \omega_{i}= \begin{cases}\frac{-b+\sqrt{b^{2}-4 a c}}{2 a} & \text { if } b \geq 0 \\ \frac{-b-\sqrt{b^{2}-4 a c}}{2 a} & \text { if } b<0\end{cases}
$$

For $a=0$, the solution coincides with a first degree Taylor approximation:

$$
\Delta \omega_{i}=-\frac{c}{b}=\Delta C t R_{i}\left[\frac{\omega_{i} \sigma_{i}^{2}}{\sigma_{\Delta B}^{2}}\left(1-2 \rho_{\Delta B, \Delta S_{i}}^{2}\right)+\frac{\sigma_{i} \rho_{\Delta B, \Delta S_{i}}}{\sigma_{\Delta B}}\right]^{-1}
$$

\section{A.2. THE ERC ALGORITHM}

We know that the $\omega_{E R C}$ basket must verify that all Contributions to Risk are equal: $C t R_{i}=\frac{1}{n}, \forall i$. Thus, for any $i$ we can compute the step size $\Delta \omega_{i}$ so that any deviation from that value, $\Delta C t R_{i}=$ $\frac{1}{n}-C t R_{i}$, could be corrected through the expression in Eq. (36) (if $a \neq 0$ ) or Eq. (37) (if $a=0$ ). The following algorithm computes the $\left\{C t R_{i}\right\}$ vector, determines for which leg $i$ the deviation $\left|\Delta C t R_{i}\right|$ is greatest, and computes the corresponding $\Delta \omega_{i}$ that reduces such deviation in the next iteration. The algorithm stops when either the desired accuracy has been achieved, or iterations exceed a user-designated limit. Figures 14 and 15 show how holdings converge to their optimal values for the examples of ERC hedging and trading baskets discussed in Section 3 and 4. 


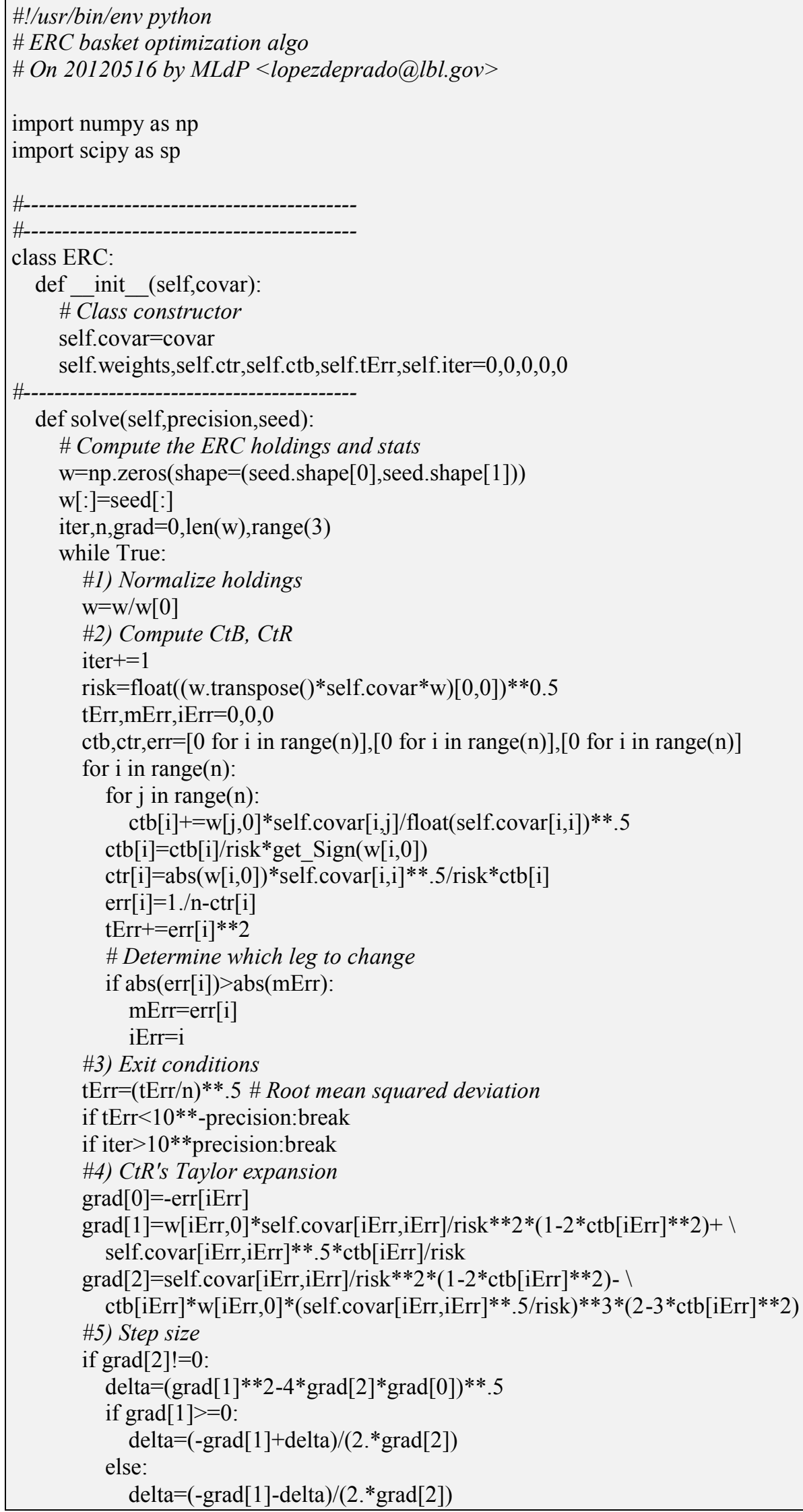




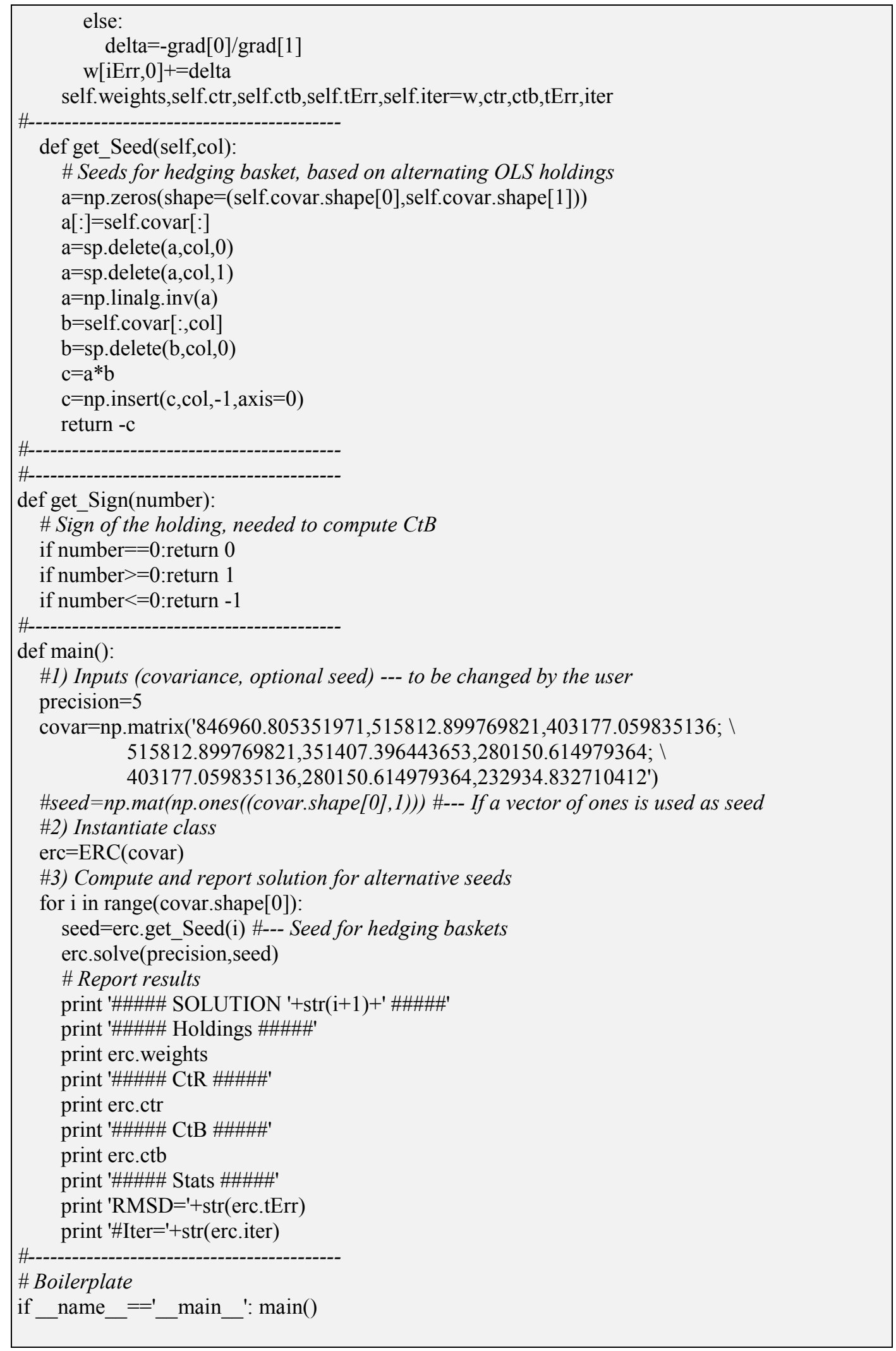

[FIGURE 14 HERE] 
[FIGURE 15 HERE]

\section{A.3. COMPUTING THE MMSC BASKETS FOR $\boldsymbol{n}$-INSTRUMENTS}

What follows is a synthesis of some of the results derived in López de Prado and Leinweber (2012). Please refer the reader to that publication for the detailed proofs.

For any two subsets $i$ and $j$, we can define the following variables:

$$
\begin{gathered}
a=\frac{1}{2} \frac{\sigma_{\Delta S_{i}}^{2}}{\sigma_{\Delta B}^{2}}\left[\rho_{\Delta B, \Delta S_{j}}+\rho_{\Delta B, \Delta S_{i}}\left(2 \rho_{\Delta S_{i}, \Delta S_{j}}-3 \rho_{\Delta B, \Delta S_{j}} \rho_{\Delta B, \Delta S_{i}}\right)\right] \\
b=-\frac{\sigma_{\Delta S_{i}}}{\sigma_{\Delta B}}\left(\rho_{\Delta S_{i}, \Delta S_{j}}-\rho_{\Delta B, \Delta S_{j}} \rho_{\Delta B, \Delta S_{i}}\right) \\
c=\Delta \rho_{\Delta B, \Delta S_{j}}
\end{gathered}
$$

where $i=1, \ldots, N$ subsets, $N=\sum_{i=1}^{n-1}\left(\begin{array}{c}n \\ i\end{array}\right)=2\left(2^{n-1}-1\right)$ is the number of subsets, $\Delta S_{i}=$ $\sum_{j=1}^{n} \Delta P_{j} \breve{\omega}_{i, j}$, and $\breve{\omega}_{i}$ is the vector of holdings of subset $i$. Then, for $a \neq 0$ :

$$
\delta_{i}= \begin{cases}\frac{-b+\sqrt{b^{2}-4 a c}}{2 a} & \text { if } b \geq 0 \\ \frac{-b-\sqrt{b^{2}-4 a c}}{2 a} & \text { if } b<0\end{cases}
$$

And for $a=0$ :

$$
\delta_{i}=-\frac{c}{b}=\Delta \rho_{\Delta B, \Delta S_{i}} \frac{\sigma_{\Delta B}}{\sigma_{\Delta S_{i}}\left(\rho_{\Delta S_{i}, \Delta S_{j}}-\rho_{\Delta B, \Delta S_{j}} \rho_{\Delta B, \Delta S_{i}}\right)}
$$

This result allows us to compute the step size $\delta_{i}$ for subset $i$ that will change the correlation between subset $j$ and the basket, $\Delta \rho_{\Delta B, \Delta S_{j}}$. This change in subset $i$ can be backpropagated to the legs involved in that subset by multiplying the holdings of the legs that form subset $i$ by $(1+$ $\left.\delta_{i}\right)$. For example, a change in subset $i$ is backpropagated by setting the leg's holdings to $\omega_{j}=$ $\breve{\omega}_{i, j}\left(1+\delta_{i}\right), j=1, \ldots, n$, for the next iteration (see function get_Backpropagate in Section A.4). By doing so, we can balance the exposure to a subset $j$, even if it is composed of instruments that are not tradable or subject to constraints.

\section{A.4. THE MMSC ALGORITHM}

The hedging basket is determined by minimizing the maximum $\rho_{\Delta B, \Delta S_{i}}$, where $i=1, \ldots, N$ represents any of the $N$ subsets of legs (including the $n$ legs). In each iteration, we identify the subset $j$ for which $\Delta \rho_{\Delta B, \Delta S_{j}}=\frac{1}{N}\left(\sum_{i=1}^{N} \rho_{\Delta B, \Delta S_{i}}\right)-\rho_{\Delta B, \Delta S_{j}}$ is lowest. This is the subset whose correlation we would like to bring down to the average value. This can be done by changing the 
holdings of that subset $j$ or, if it is not tradable or its holding is constrained, by changing any other subset's holdings $i$. By reducing our exposure to the subsets with above average correlations, we bring that average down until there is no possibility to keep reducing $\rho_{\Delta B, \Delta S_{j}}$ without producing some other $\rho_{\Delta B, \Delta S_{i}}>\rho_{\Delta B, \Delta S_{j}}$, at which point the solution has been found.

For a trading basket, the algorithm is essentially the same, with the only difference that at each iteration we identify the subset $j$ for which $\Delta \rho_{\Delta B, \Delta S_{j}}=\frac{1}{N}\left(\sum_{i=1}^{N} \rho_{\Delta B, \Delta S_{i}}\right)-\rho_{\Delta B, \Delta S_{j}}$ is highest. This Maximizes the Minimum Subset Correlation (the purpose of a trading basket), as opposed to Minimize the Maximum Subset Correlation (the goal of a hedging basket).

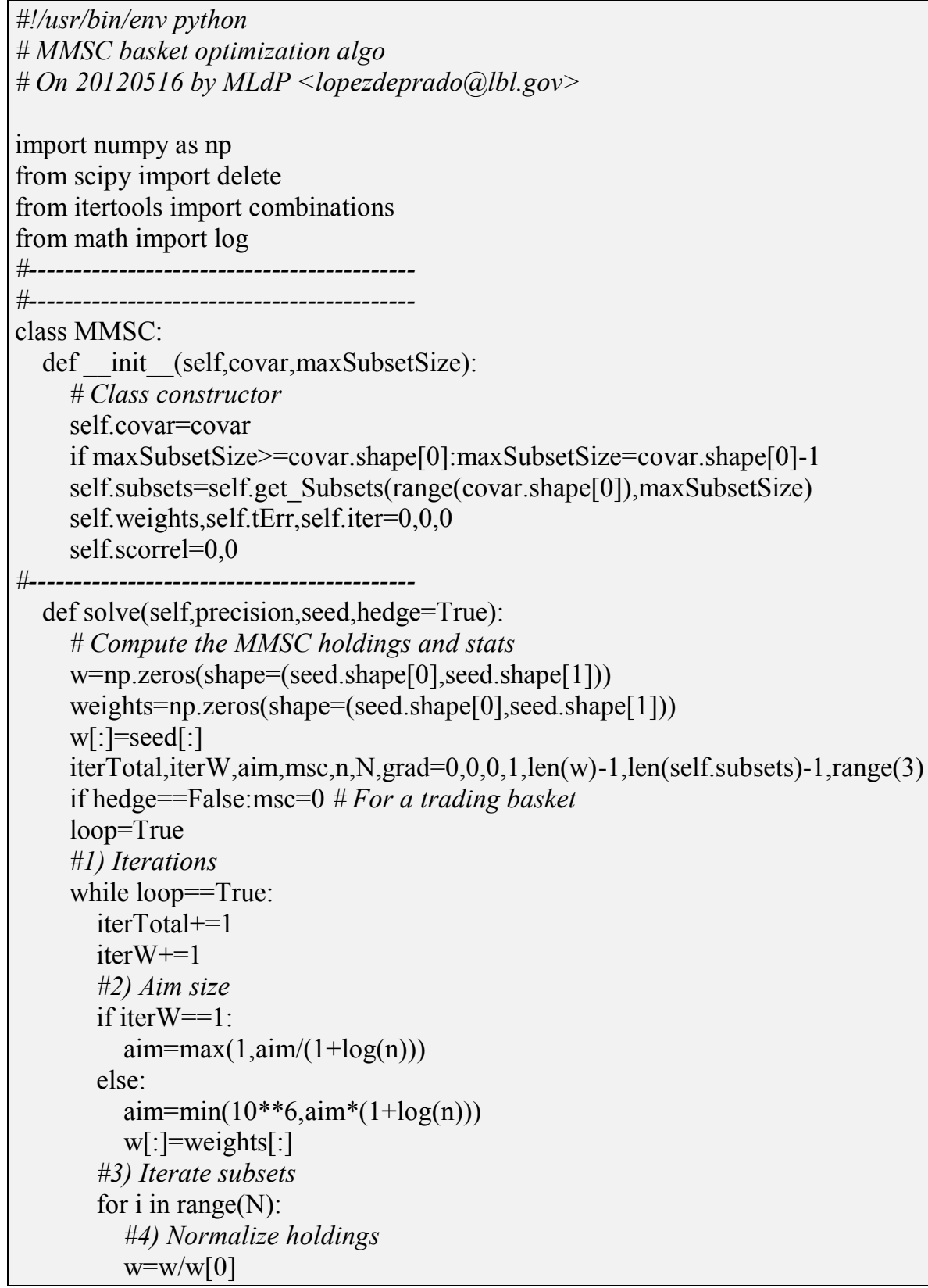




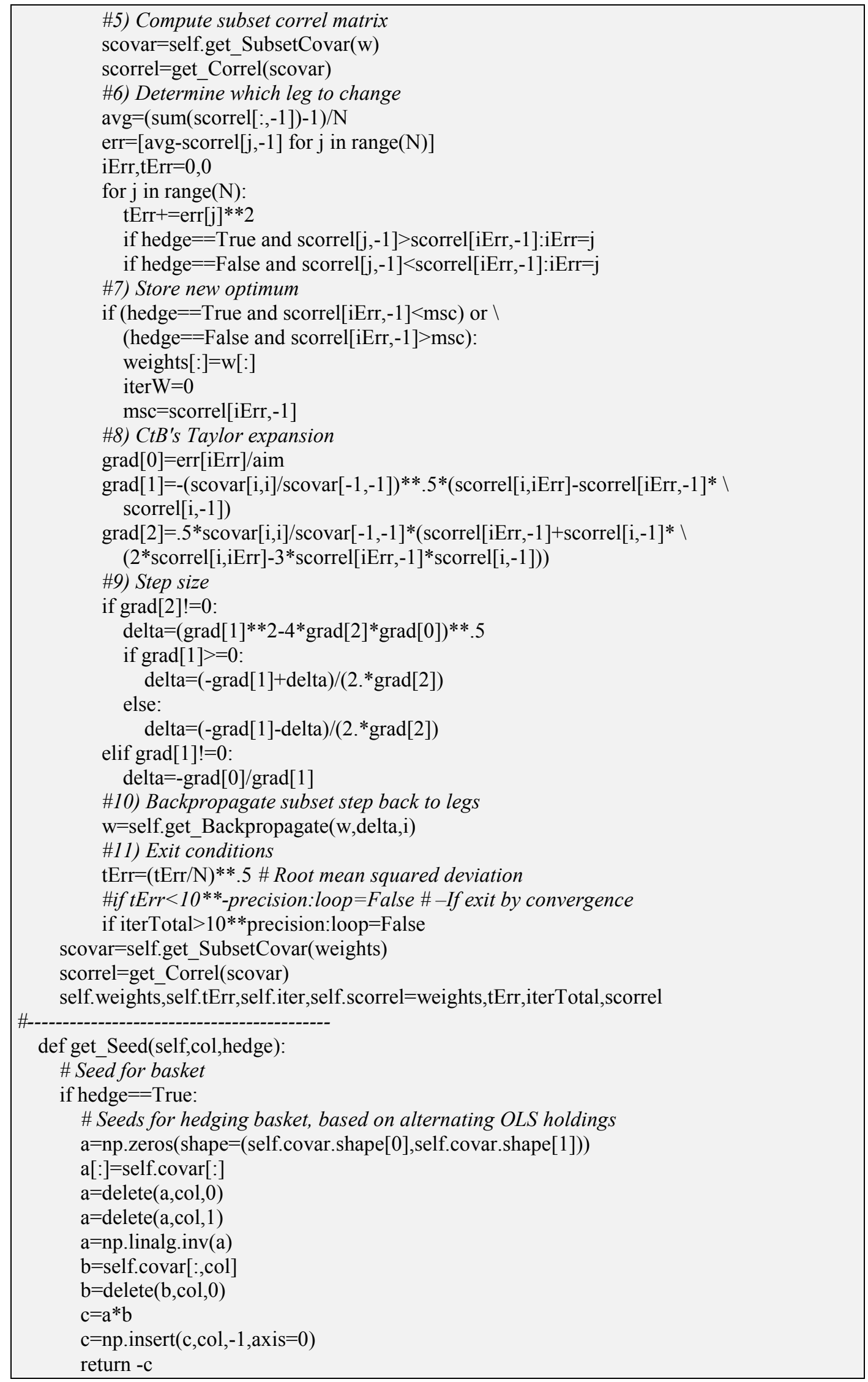




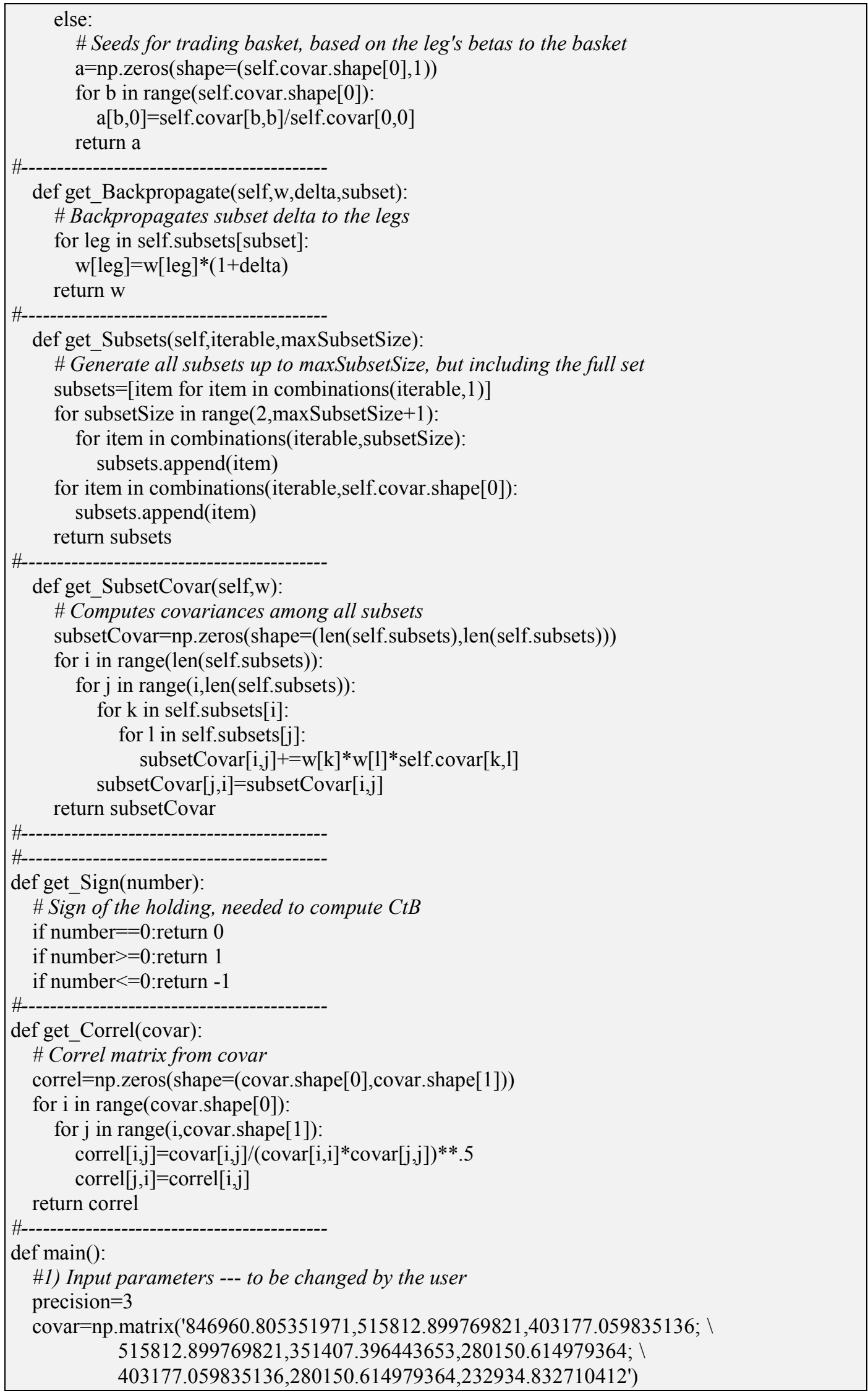




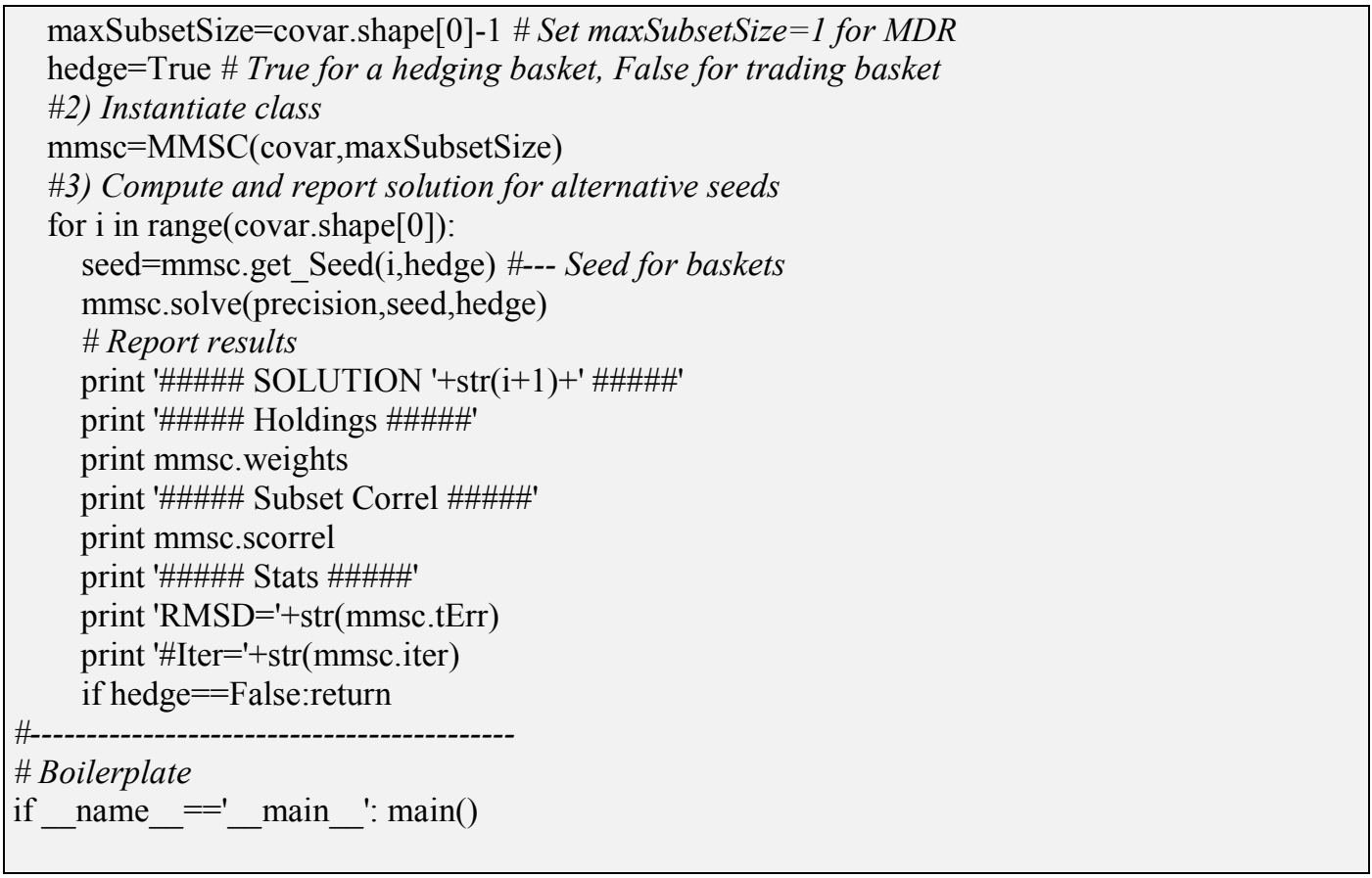

Figures 16 and 17 show how holdings converge to their optimal values for the examples of MMSC hedging and trading baskets discussed in Section 3 and 4.

\section{[FIGURE 16 HERE]}

[FIGURE 17 HERE]

This algorithm can also be used to compute the MDR solution, by setting the parameter maxSubsetSize $=1$. This is equivalent to a MMSC optimization where subsets of more than one leg are ignored. maxSubsetSize can also be used to skip evaluating correlations for subsets of larger size, which is convenient should $N$ reach an impracticable order of magnitude.

\section{A.5. COVARIANCE CLUSTERING}

The number of subsets follows a power law on the number $n$ of instruments involved: $N=$ $\sum_{i=1}^{n-1}\left(\begin{array}{c}n \\ i\end{array}\right)=2\left(2^{n-1}-1\right)$, where we exclude the empty set and the full set. For a sufficiently large $n$, the number of subsets $N$ to be evaluated per iteration makes MMSC impracticable. But because $n$ is large, it also becomes more likely that some of the instruments involved are highly correlated. An approach commonly used to reduce the dimension of a problem applies PCA to identify which orthogonal directions add least variance, so that they can be dropped. There are at least three arguments for discarding such procedure in the context of balanced baskets: First, a key reason for favoring balanced baskets was precisely that they did not require a change of basis, so that the solution could be intuitively connected to the original instruments and subsets of them. Second, "dropping" dimensions involves the loss of information, even if minimal. Third, in a capital allocation context, we cannot short funds or portfolio managers. 
In this section we propose a new method for reducing the dimension of a covariance matrix without requiring a change of basis or dropping dimensions. The intuition is to identify which tuples of matrix columns point in neighboring directions, in which case they are redundant and can be clustered together. This can be evaluated by carrying out an eigen-decomposition of the matrix and evaluating which columns have the largest loadings in the orthogonal directions that contribute least variance. When such tuples are clustered together they form a new column that is less redundant, thus contributing to a more parsimonious distribution of the variance across the orthogonal directions. The actual clustering is done by recursively aggregating any two columns $(i, j)$, applying the property that

$$
\begin{aligned}
& \sigma_{i+j, k}=E[(i+j-E[i+j])(k-E[k])] \\
& \quad=E[(i-E[i]+j-E[j])(k-E[k])] \\
& =E[(i-E[i])(k-E[k])+(j-E[j])(k-E[k])] \\
& \quad=\sigma_{i, k}+\sigma_{j, k} \\
& \sigma_{i+j, i+j}=\sigma_{i, i+j}+\sigma_{j, i+j}=\sigma_{i, i}+\sigma_{j, j}+2 \sigma_{i, j}
\end{aligned}
$$

The algorithm identifies what column aggregation minimizes the matrix's condition number at each iteration. In this way, we reduce $n$ and, more importantly, $N$, while making the covariance matrix less singular. One possible procedure would consist in computing, by brute force, all possible clustering outcomes, and determine the one for which the condition number is minimal. That would require $\sum_{i=1}^{m<n}\left(\begin{array}{c}n \\ i\end{array}\right)$ covariance clustering operations, where $m$ is the number of dimensions reduced. This is a very large number, considering that the reason for clustering was to avoid having to evaluate $N$ subset correlations per iteration. Applying brute force does not seem to alleviate our computational problem. An alternative clustering strategy would consist in sequentially pairing columns of the covariance matrix, so that at each iteration we minimize the condition number. That strategy only requires $\sum_{i=1}^{m<n}\left(\begin{array}{c}n-i+1 \\ 2\end{array}\right) \ll \sum_{i=1}^{m<n}\left(\begin{array}{c}n \\ i\end{array}\right)$ covariance clustering operations.

More precisely, suppose a covariance matrix $V$ with elements $\sigma_{i, j}$ and $i, j=1, \ldots, n$. We denote the matrix's $i$-th eigenvalue by $\lambda_{i}$, and its condition number by $c=\frac{\max _{i}\left\{\lambda_{i}\right\}}{\min _{i}\left\{\lambda_{i}\right\}}=\frac{\lambda_{1}}{\lambda_{N}}$. We would like to reduce $V$ 's order to a more manageable $n^{*}=n-m$, where $2 \leq n^{*}<n$. The following algorithm clusters $m$ elements of $V$ until such requirement is met:

1. If $n=n^{*}$, return $V$ and exit.

2. For each pair $(i, j), i<j$ of columns of $V$,

a. Let $\tilde{V}=V$ be a copy of $V$.

b. Insert in $\tilde{V}$

i. column and row, $n+1$, with $k$ elements, $\sigma_{k, n+1}=\sigma_{k, i}+\sigma_{k, j}, \forall k$.

ii. diagonal element $\sigma_{n+1, n+1}=\sigma_{i, n+1}+\sigma_{j, n+1}=\sigma_{i, i}+\sigma_{j, j}+2 \sigma_{i, j}$.

c. Strike down columns and rows $i, j$, giving $\tilde{V}$ an order $\tilde{n}=n-1$.

d. Compute $\left\{\tilde{\lambda}_{l}\right\}$ for the resulting $\tilde{V}$. 
e. Store the value $c_{i, j}=\frac{\max _{l}\left\{\tilde{\lambda_{l}}\right\}}{\min _{l}\left\{\widetilde{\lambda_{l}}\right\}}=\frac{\widetilde{\lambda_{1}}}{\widetilde{\lambda_{N}}}$.

3. Determine the $\left(i^{*}, j^{*}\right)=\arg \max i<j \quad c_{i, j}$

4. Prepare for the next iteration

$$
1<j \leq n
$$

a. Replace $V$ with the matrix $\widetilde{V}$ which clustered together elements $\left(i^{*}, j^{*}\right)$.

b. Set $n=\tilde{n}$.

5. Loop to 1 .

The outcome is a clustered covariance matrix with three crucial properties:

1. It has a smaller dimension, which enables calculations to be carried out in a feasible amount of time.

2. It has a smaller condition number, and consequently it is less singular. Financial applications typically require the inversion of the covariance matrix, and near-singular covariance matrices are a major source of numerically unstable results.

3. This clustering of the covariance matrix forms a disjoint-set data structure, whereby each original element will end up in only one cluster. Cluster constituents are equally (and positively) weighted, thus the sum of elements of the covariance matrix is kept constant across iterations. This is a key difference with respect to PCA, where each constituent forms part of each component, and weights are allowed to be negative.

\section{[FIGURE 18 HERE]}

A numerical example with illustrate how the algorithm works. Suppose that we are given a covariance matrix of the 88 most liquid futures contracts. Computing a MMSC hedging basket of that dimension seems quite impracticable. Even though no column may be derived as an exact linear combination of the rest, it is very likely that one column spans a vector in a very close angle to another column or a combination of columns. The algorithm above will identify those situations and form clusters of very similar instruments. Figure 18 shows how, beginning with a numerically ill-conditioned covariance matrix of 88 instruments (determinant greater than $10^{305}$, condition number of 486,546.1293), the covariance matrix is greatly improved by clustering. For example, for $n^{*}=20$, the condition number has dropped to a fraction of its original value (677.41739). As a result, the covariance matrix will now be less numerically ill-conditioned, and $n$ can be reduced to a value for which the MMSC solution can be computed.

We believe this algorithm will prove useful in many financial applications beyond basket construction, like in capital allocation and portfolio optimization problems. What follows is an implementation in Python code. The user simply needs to adjust the statement path='E: $\backslash H F T \backslash$ Covariance.csv' with the path where a covariance matrix is stored in $\operatorname{csv}$ format.

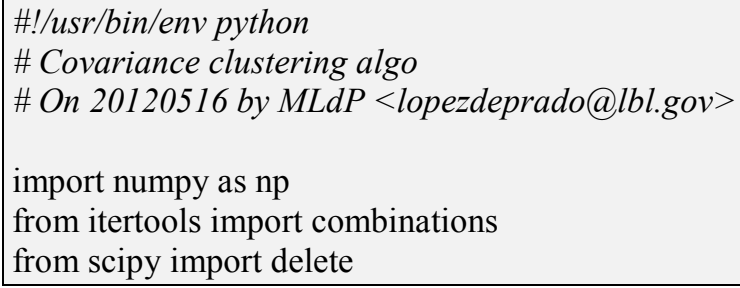




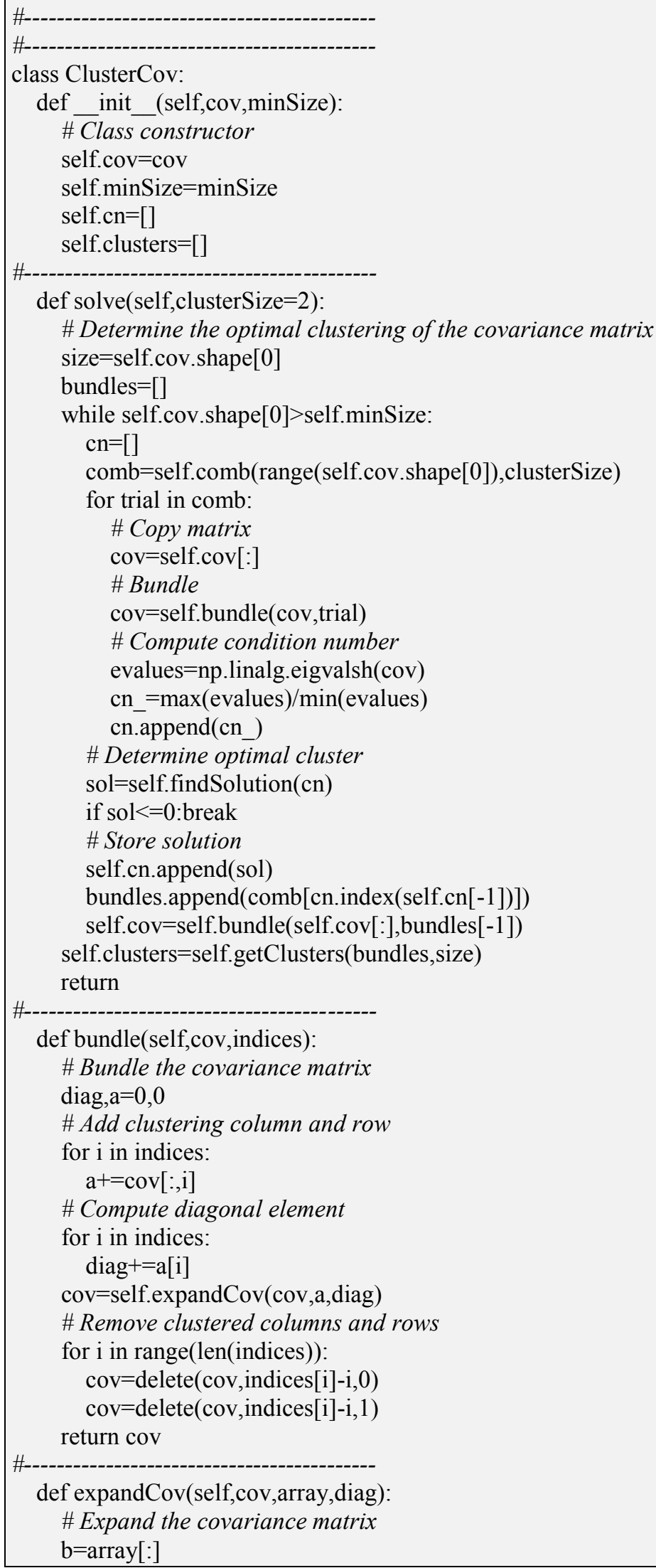




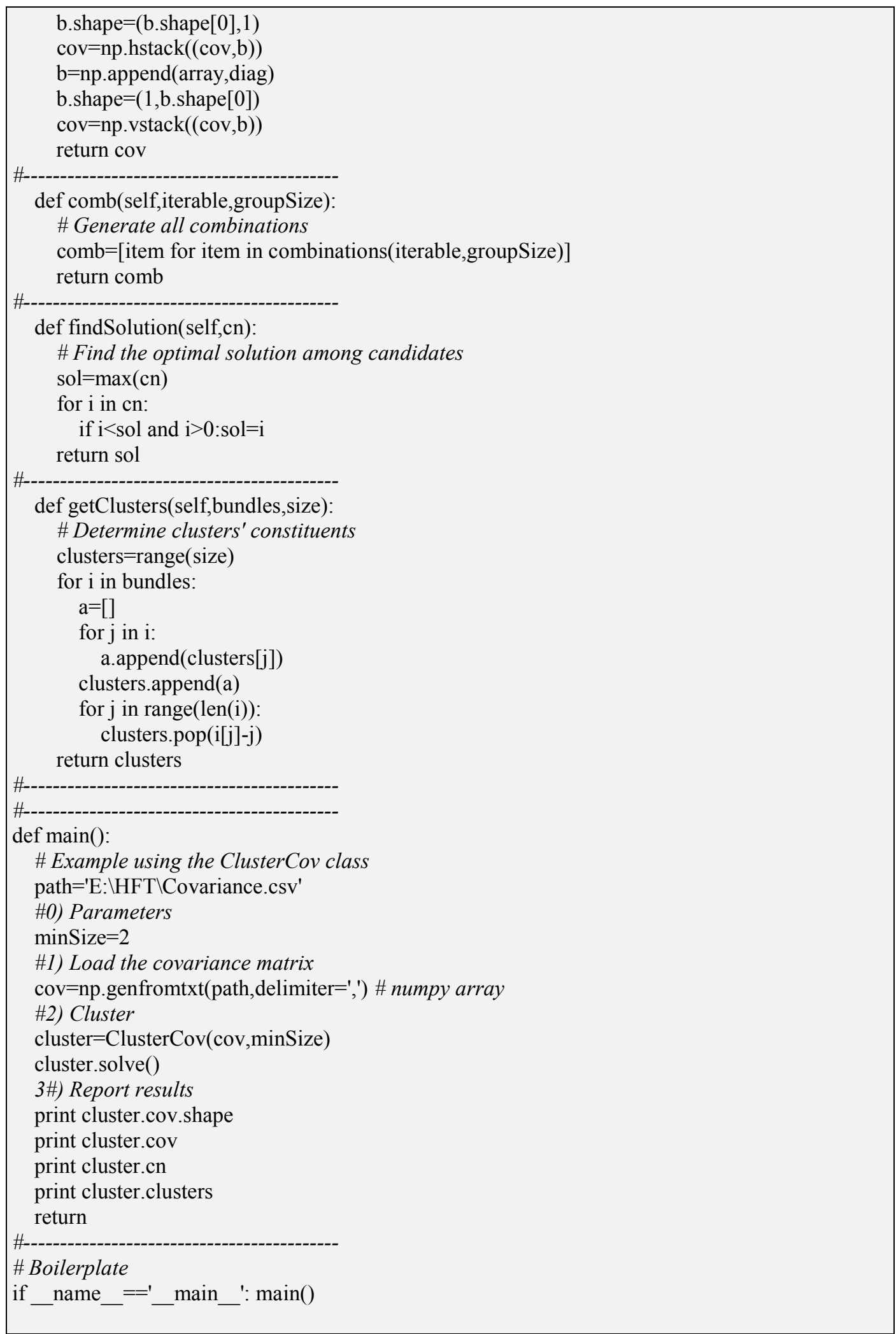




\section{FIGURES}

\begin{tabular}{|c|c|c|c|c|c|}
\hline & FA1 Index & ES1 Index & & FA1 Index & ES1 Index \\
\hline FA1 Index & 846960.8 & 515812.9 & FA1 Index & 1.000000 & 0.945486 \\
\hline ES1 Index & 515812.9 & 351407.4 & ES1 Index & 0.945486 & 1.000000 \\
\hline
\end{tabular}

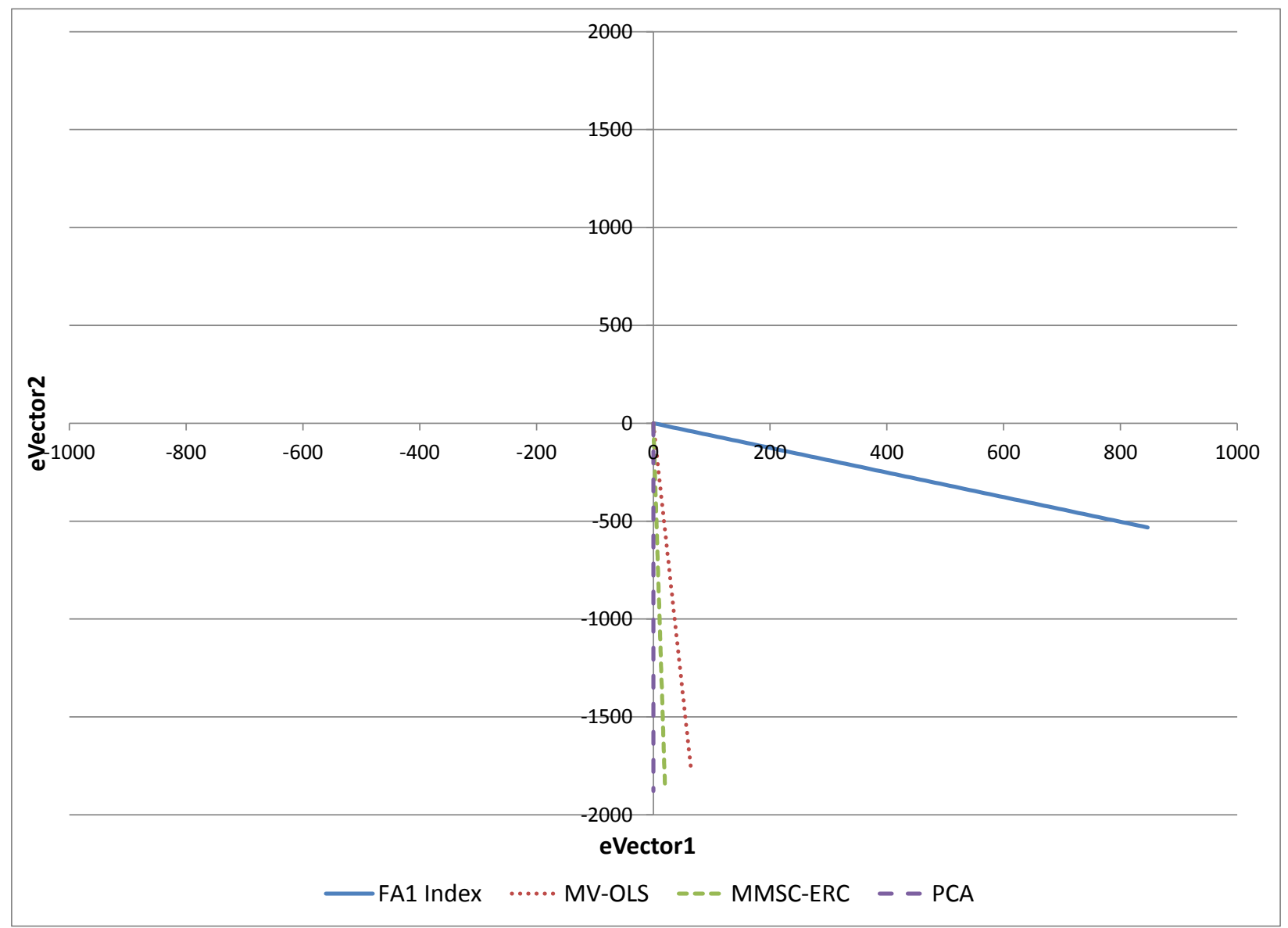

Figure 2 - Graphical interpretation of the alternative hedging baskets

We can plot the original position $\left(h_{1}\right)$ as a vector in a two-dimensional space characterized by the eigen-decomposition of the covariance matrix from Figure 1. This vector lays in a direction very close to the first eigenvector, which is associated with the market risk component. If we hedge "FA1 Index" with "ES1 Index" using the MV or OLS procedures, the basket's vector spans in a direction very close to the second eigenvector, which is associated with the spread risk. The MMSC and ERC procedures give a solution very similar to PCA's without requiring a basis change. 


\begin{tabular}{|c|c|c|c|c|c|c|c|}
\hline & FA1 Index & ES1 Index & DM1 Index & & FA1 Index & ES1 Index & DM1 Index \\
\hline FA1 Index & 846960.8 & 515812.9 & 403177.1 & FA1 Index & 1.000000 & 0.945486 & 0.907710 \\
\hline ES1 Index & 515812.9 & 351407.4 & 280150.6 & ES1 Index & 0.945486 & 1.000000 & 0.979194 \\
\hline \multirow[t]{2}{*}{ DM1 Index } & 403177.1 & 280150.6 & 232934.8 & DM1 Index & 0.907710 & 0.979194 & 1.000000 \\
\hline & FA1 Index & ES1 Index & DM1 Index & & FA1 Index & ES1 Index & DM1 Index \\
\hline FA1 Index & 0.774883836 & -0.62081023 & 0.118952497 & FA1 Index & 1381000.26 & 0 & 0 \\
\hline ES1 Index & 0.495126356 & 0.479137368 & -0.72476015 & ES1 Index & 0 & 45883.18712 & 0 \\
\hline DM1 Index & 0.39294393 & 0.620501441 & 0.67865531 & DM1 Index & 0 & 0 & 4419.589178 \\
\hline
\end{tabular}

Figure 3 -Covariance and Correlation matrices for the proposed hedging problem (three instruments), with the corresponding eigenvectors and eigenvalues matrices

\begin{tabular}{|c|c|c|c|c|c|}
\hline & \multicolumn{4}{|c|}{ ERC } & \\
\hline & INSTRUMENTS & HOLDINGS & CtB & CtR & \\
\hline & FA1 Index & 1000 & 0.14 & 0.33 & \\
\hline & ES1 Index & -4110 & 0.05 & 0.33 & \\
\hline & DM1 Index & 3274 & 0.08 & 0.33 & \\
\hline Subsets & 2 & 3 & 1,2 & 1,3 & 2,3 \\
\hline $1,2,3$ & $0.135702 \quad 0.0512$ & 510.079026 & 0.156600 & 0.102101 & 0.264190 \\
\hline
\end{tabular}

Figure 4-One possible ERC solution

The second table in Figure 4 reports the correlation of the basket to each subset of legs, where the subset is identified by the legs it is made of. For example, subset $(1,3)$ is composed of "ES1 Index" and "DM1 Index".

\begin{tabular}{|c|c|c|c|c|c|}
\hline & \multicolumn{4}{|c|}{ ERC } & \\
\hline & INSTRUMENTS & HOLDINGS & CtB & CtR & \\
\hline & FA1 Index & 1000 & 0.97 & 0.33 & \\
\hline & ES1 Index & 1515 & 0.99 & 0.33 & \\
\hline & DM1 Index & 1885 & 0.98 & 0.33 & \\
\hline Subsets & 2 & 3 & 1,2 & 1,3 & 2,3 \\
\hline $1,2,3$ & $0.969597 \quad 0.9934$ & $148 \quad 0.980552$ & 0.995030 & 0.998350 & 0.992132 \\
\hline
\end{tabular}

Figure 5-An alternative ERC solution 


\begin{tabular}{|c|c|c|c|c|c|}
\hline & \multicolumn{4}{|c|}{ DRP } & \\
\hline & INSTRUMENTS & HOLDINGS & CtB & CtR & \\
\hline & FA1 Index & 1000 & -0.49 & -0.12 & \\
\hline & ES1 Index & 18338 & -0.62 & -1.75 & \\
\hline & DM1 Index & -29896 & 0.77 & 2.87 & \\
\hline Subsets & 2 & 3 & 1,2 & 1,3 & 2,3 \\
\hline $1,2,3$ & $\begin{array}{lll}-0.492806 & -0.6197\end{array}$ & 240.765364 & -0.612224 & 0.778720 & 0.983147 \\
\hline
\end{tabular}

Although the DRP method is not a balanced basket approach, we report its solution here to illustrate the point that procedures which require a change of basis may yield rather unintuitive solutions.

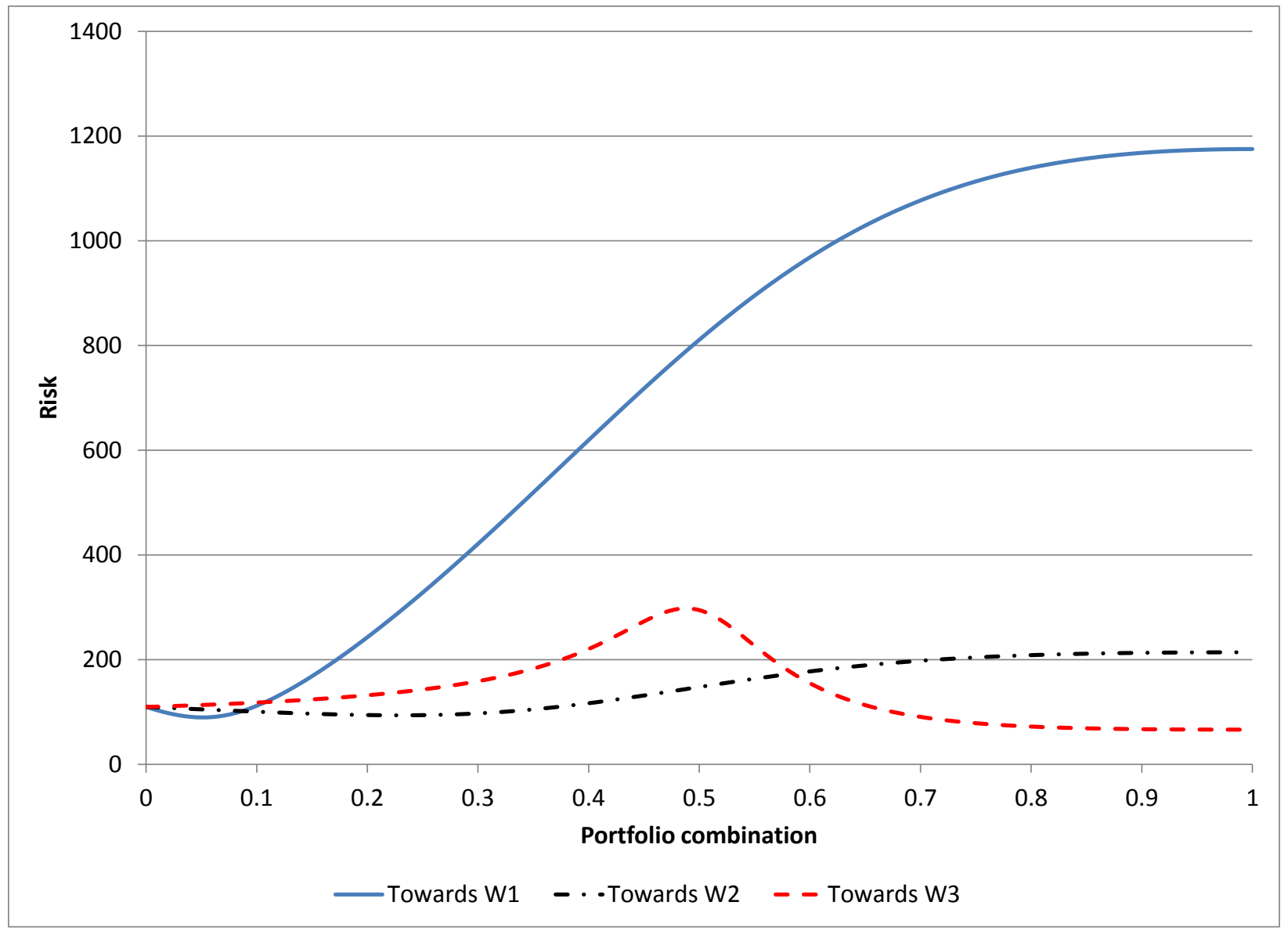

Figure 7(a) - Risk as we move away from DRP and towards the eigenvectors

Risk increases as we approach a basket pointing in the direction of an eigenvector, except in the case of the eigenvector associated with the lowest eigenvalue. This is because eigenvectors are the critical points of the Rayleigh quotient $\frac{\omega^{\prime} V \omega}{\omega^{\prime} \omega}$, where the numerator is the variance of the basket. 


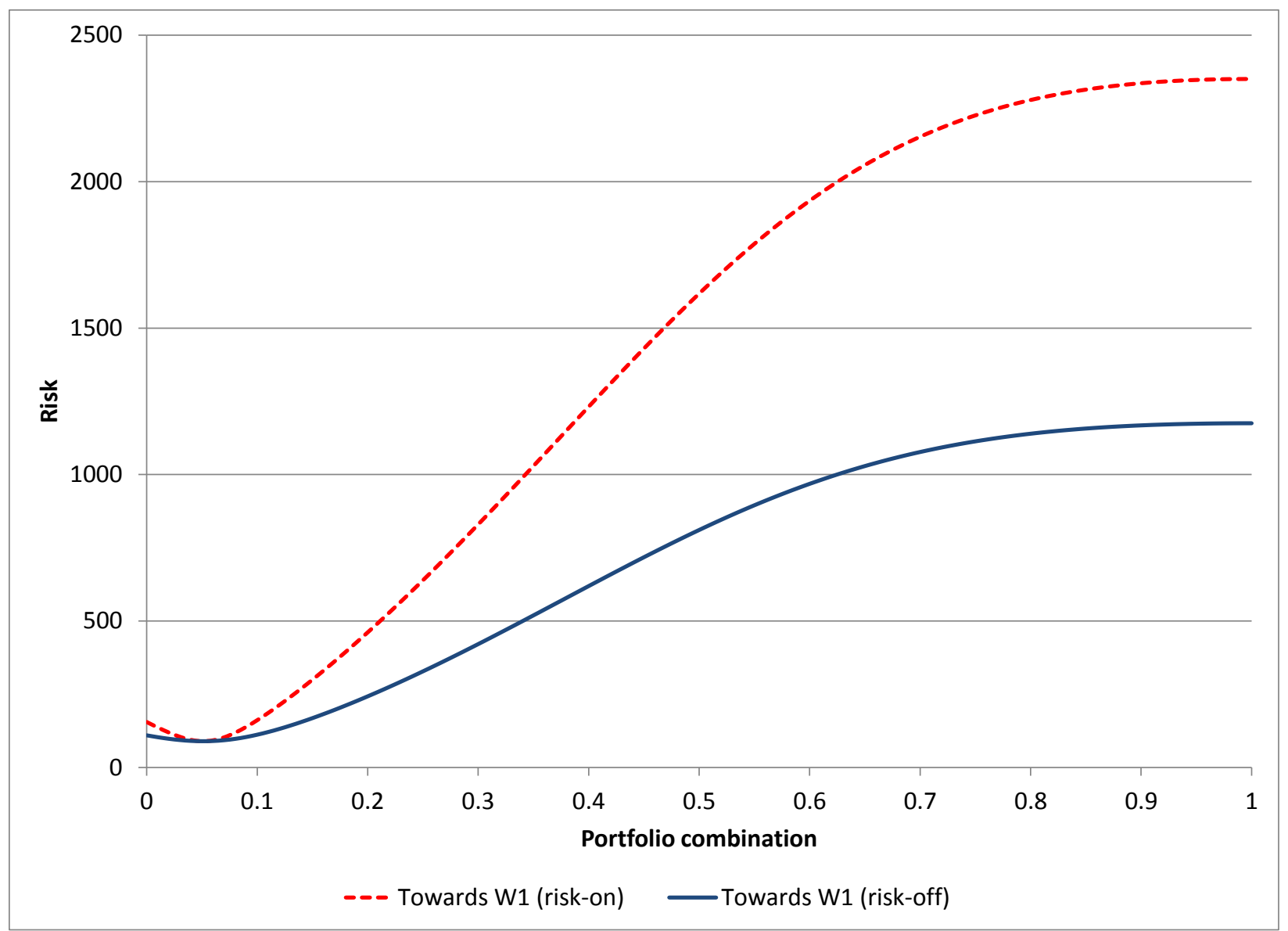

Figure 7(b) - Risk as we move away from DRP and towards the first eigenvector, in a risk-on and risk-off environment

\begin{tabular}{|c|c|c|c|c|c|}
\hline & \multicolumn{4}{|c|}{ MDR } & \\
\hline & INSTRUMENTS & HOLDINGS & $\mathrm{CtB}$ & CtR & \\
\hline & FA1 Index & 1000 & 0.08 & 0.16 & \\
\hline & ES1 Index & -4736 & 0.08 & 0.49 & \\
\hline & DM1 Index & 4031 & 0.08 & 0.34 & \\
\hline Subsets & 2 & 3 & 1,2 & 1,3 & 2,3 \\
\hline $1,2,3$ & $0.075176 \quad 0.0751$ & $\begin{array}{ll}76 & 0.075176\end{array}$ & 0.142953 & 0.076735 & 0.362830 \\
\hline
\end{tabular}

Figure 8 - The MDR solution 


\begin{tabular}{|c|c|c|c|c|c|}
\hline & \multicolumn{4}{|c|}{ MMSC } & \\
\hline & INSTRUMENTS & HOLDINGS & CtB & CtR & \\
\hline & FA1 Index & 1000 & 0.19 & 0.50 & \\
\hline & ES1 Index & -3632 & 0.03 & 0.22 & \\
\hline & DM1 Index & 2690 & 0.07 & 0.28 & \\
\hline Subsets & 2 & 3 & 1,2 & 1,3 & 2,3 \\
\hline $1,2,3$ & $0.185136 \quad 0.0341$ & $164 \quad 0.074562$ & 0.185136 & 0.123218 & 0.185136 \\
\hline
\end{tabular}

Figure 9-The MMSC solution

\begin{tabular}{|c|ccc|c|}
\hline F1 & ERC & MDR & MMSC & Min Exposure \\
\hline $\mathbf{1}$ & 774.88384 & 774.88384 & 774.88384 & 774.88384 \\
$\mathbf{2}$ & -2035.20002 & -2344.85515 & -1798.47923 & -1798.47923 \\
$\mathbf{3}$ & 1286.55426 & 1584.11666 & 1057.18793 & 1057.18793 \\
$\mathbf{1 , 2}$ & -1260.31618 & -1569.97131 & -1023.59540 & -1023.59540 \\
$\mathbf{1 , 3}$ & 2061.43809 & 2359.00050 & 1832.07176 & 1832.07176 \\
$\mathbf{2 , 3}$ & -748.64576 & -760.73848 & -741.29131 & -741.29131 \\
$\mathbf{1 , 2 , 3}$ & 26.23808 & 14.14535 & 33.59253 & 14.14535 \\
\hline \hline F2 & ERC & MDR & MMSC & Min Exposure \\
\hline $\mathbf{1}$ & -620.81023 & -620.81023 & -620.81023 & -620.81023 \\
$\mathbf{2}$ & -1969.47783 & -2269.13334 & -1740.40141 & -1740.40141 \\
$\mathbf{3}$ & 2031.60988 & 2501.49347 & 1669.41536 & 1669.41536 \\
$\mathbf{1 , 2}$ & -2590.28806 & -2889.94357 & -2361.21164 & -2361.21164 \\
$\mathbf{1 , 3}$ & 1410.79965 & 1880.68323 & 1048.60513 & 1048.60513 \\
$\mathbf{2 , 3}$ & 62.13206 & 232.36012 & -70.98604 & 62.13206 \\
$\mathbf{1 , 2 , 3}$ & -558.67818 & -388.45011 & -691.79627 & -388.45011 \\
\hline \hline $\mathbf{F 3}$ & ERC & MDR & MMSC & Min Exposure \\
\hline $\mathbf{1}$ & 118.95250 & 118.95250 & 118.95250 & 118.95250 \\
$\mathbf{2}$ & 2979.10190 & 3432.37145 & 2632.59280 & 2632.59280 \\
$\mathbf{3}$ & 2222.01391 & 2735.93534 & 1825.87424 & 1825.87424 \\
$\mathbf{1 , 2}$ & 3098.05440 & 3551.32394 & 2751.54529 & 2751.54529 \\
$\mathbf{1 , 3}$ & 2340.96641 & 2854.88784 & 1944.82674 & 1944.82674 \\
$\mathbf{2 , 3}$ & 5201.11581 & 6168.30679 & 4458.46704 & 4458.46704 \\
$\mathbf{1 , 2 , 3}$ & 5320.06831 & 6287.25928 & 4577.41954 & 4577.41954 \\
\hline
\end{tabular}

Figure 10 - Factor loadings for all subsets to the three principal components 


\begin{tabular}{|c|c|c|c|c|c|c|c|}
\hline & FA1 Index & ES1 Index & DM1 Index & & FA1 Index & ES1 Index & DM1 Index \\
\hline FA1 Index & 846976.4 & 515717.6 & 403266.3 & FA1 Index & 1.000000 & 0.944523 & 0.906912 \\
\hline ES1 Index & 515717.6 & 351987.8 & 279607.2 & ES1 Index & 0.944523 & 1.000000 & 0.975424 \\
\hline DM1 Index & 403266.3 & 279607.2 & 233443.7 & DM1 Index & 0.906912 & 0.975424 & 1.000000 \\
\hline
\end{tabular}

Figure 11 - Covariance and Correlation matrices following a structural break (25\% increase of $\Lambda_{3,3}$ )

\begin{tabular}{|c|cccccc|}
\hline Subsets & $\mathbf{1}$ & $\mathbf{2}$ & $\mathbf{3}$ & $\mathbf{1 , 2}$ & $\mathbf{1 , 3}$ & $\mathbf{2 , 3}$ \\
\hline ERC & 0.124551 & 0.063642 & 0.091317 & 0.168826 & 0.105856 & 0.311802 \\
MDR & 0.069397 & 0.085319 & 0.087978 & 0.154587 & 0.083732 & 0.408664 \\
MMSC & 0.170800 & 0.047725 & 0.087045 & 0.196876 & 0.124610 & 0.231720 \\
\hline
\end{tabular}

Figure 12 - Impact of the structural break $\left(25 \%\right.$ increase of $\left.\Lambda_{3,3}\right)$ on the exposures (subset correlations) of the previously computed baskets

\begin{tabular}{|c|c|c|c|c|c|}
\hline & \multicolumn{4}{|c|}{ MMSC } & \\
\hline & INSTRUMENTS & HOLDINGS & CtB & CtR & \\
\hline & FA1 Index & 1000 & 0.98 & 0.50 & \\
\hline & ES1 Index & 0 & 0.99 & 0.00 & \\
\hline & DM1 Index & 1907 & 0.98 & 0.50 & \\
\hline Subsets & 2 & 3 & 1,2 & 1,3 & 2,3 \\
\hline $1,2,3$ & $0.976655 \quad 0.9853$ & $343 \quad 0.976655$ & 0.976655 & 1.000000 & 0.976655 \\
\hline
\end{tabular}

Figure 13 - The MMSC trading basket 


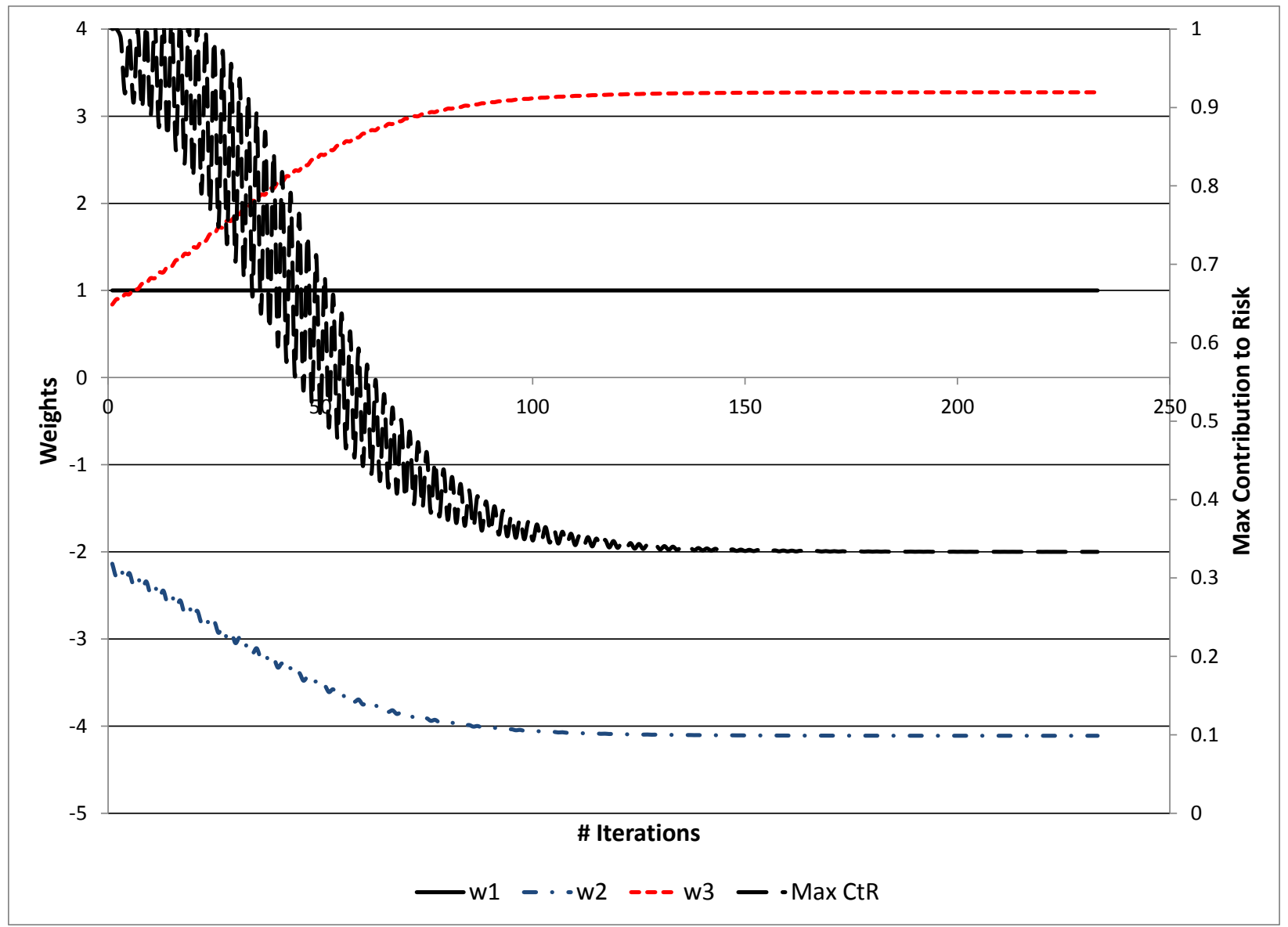

Figure 14 - Convergence of the ERC algorithm when computing the hedging basket

This figure illustrates how the ERC algorithm found the optimal hedging basket after the instruments' holdings converged over a number of iterations to a combination such that each leg contributed the same amount of risk. 


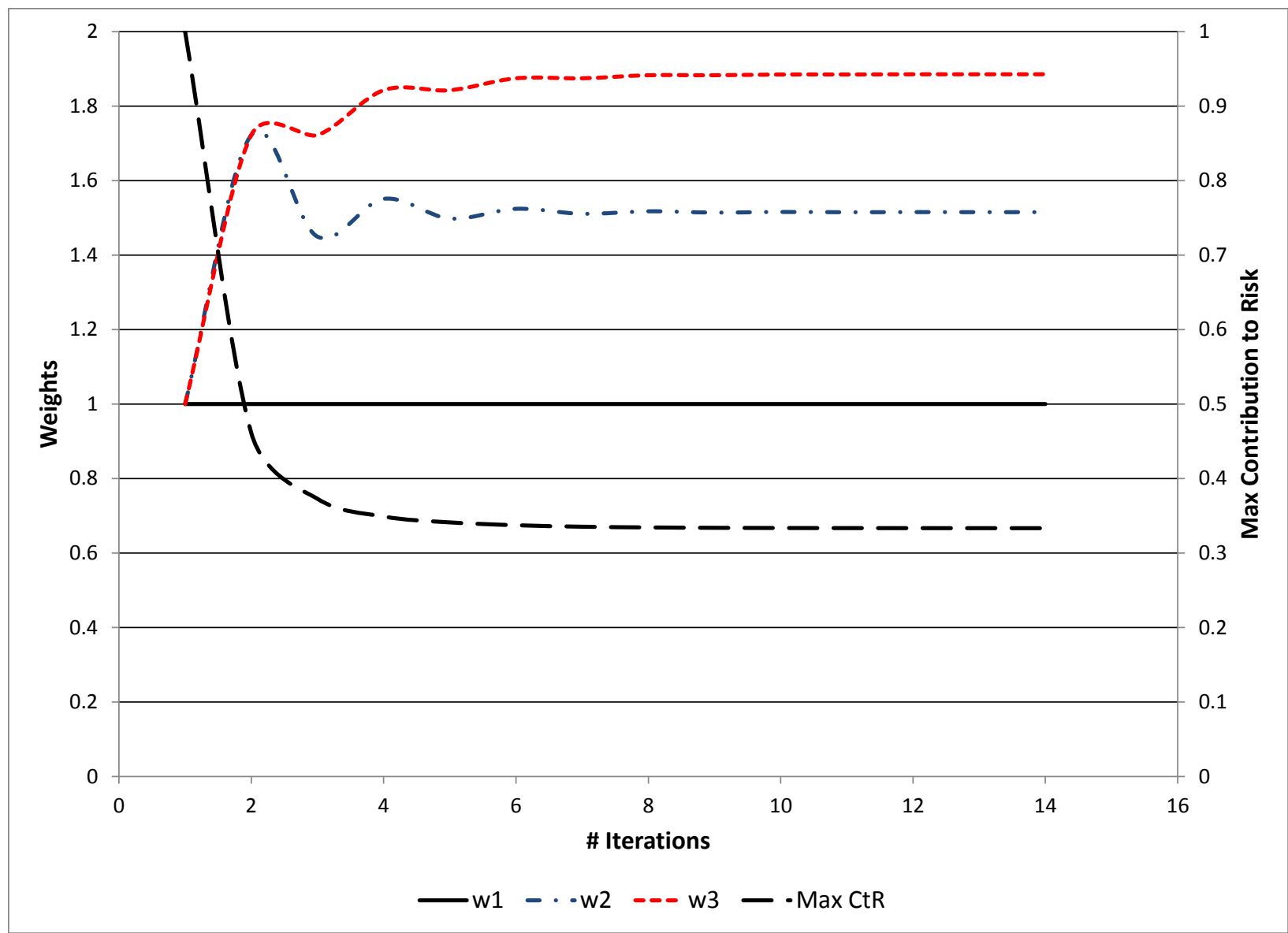

Figure 15 - Convergence of the ERC algorithm when computing the trading basket

This figure illustrates how the ERC algorithm found the optimal trading basket after the instruments' holdings converged over a number of iterations to a combination such that each leg contributed the same amount of risk. 


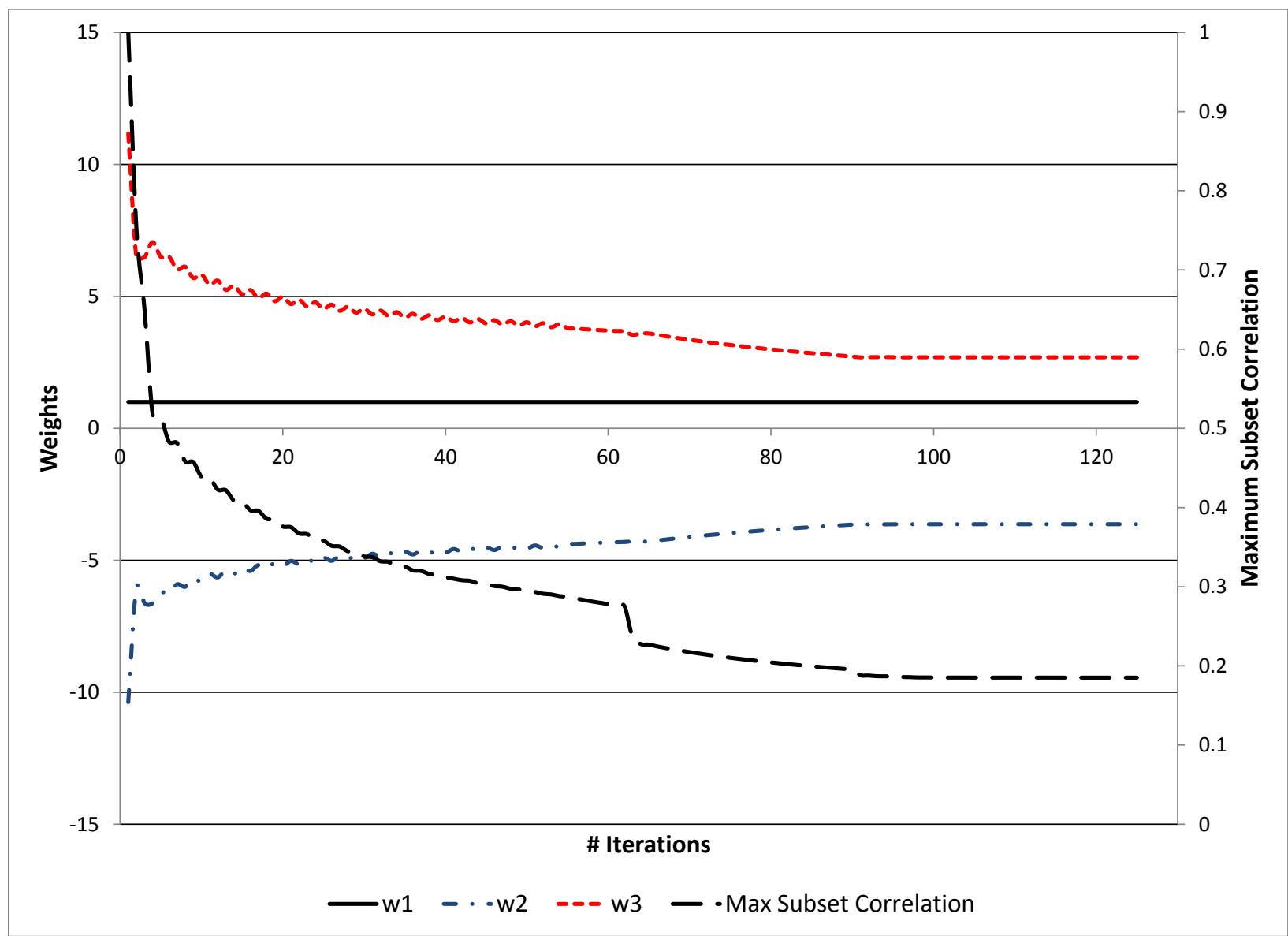

Figure 16-Convergence of the MMSC algorithm when computing the hedging basket

This figure illustrates how the MMSC algorithm found the optimal hedging basket after the instruments' holdings converged over a number of iterations to a combination that minimized the maximum subset correlation. 


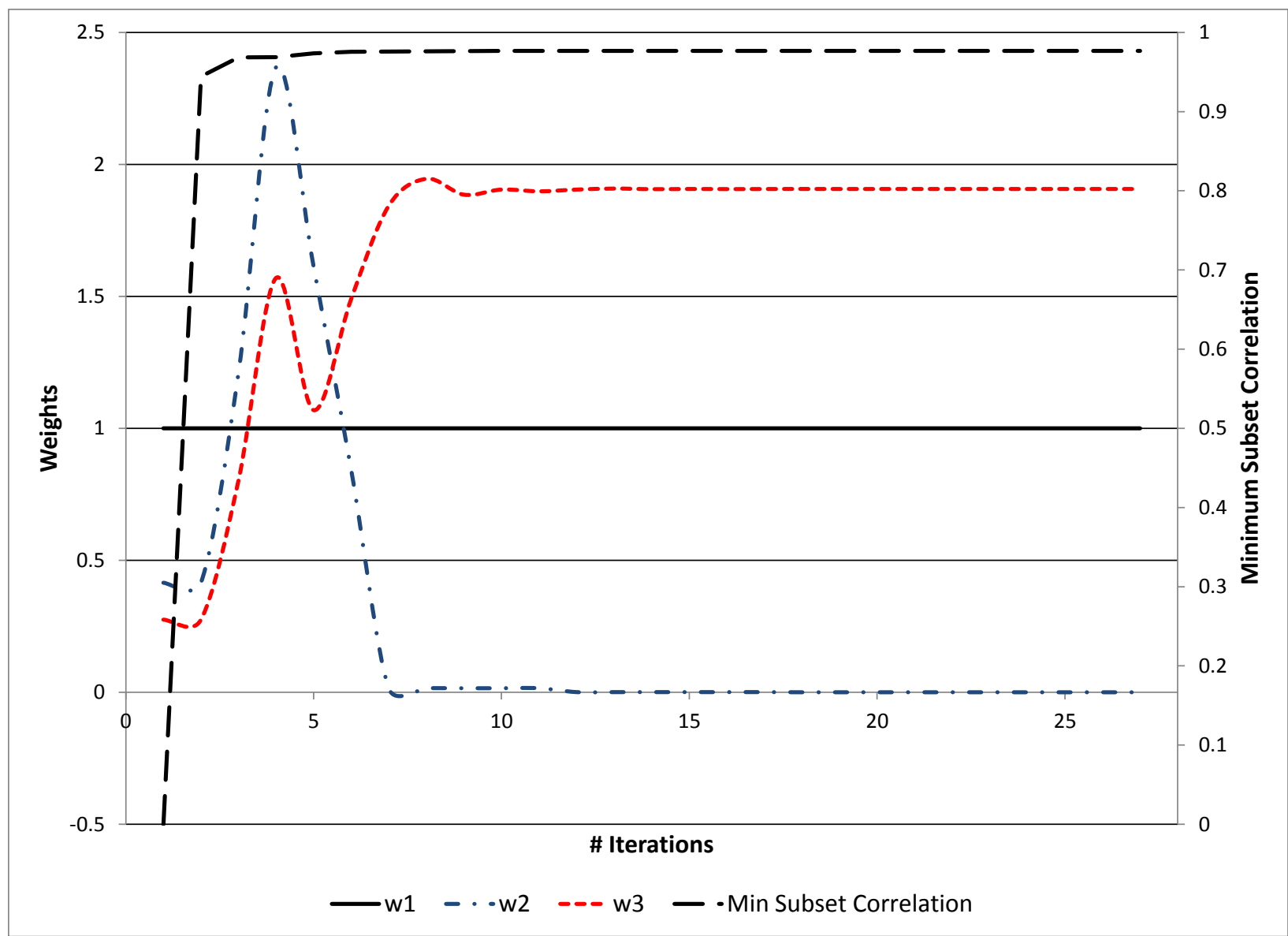

Figure 17 - Convergence of the MMSC algorithm when computing the trading basket

This figure illustrates how the MMSC algorithm found the optimal trading basket after the instruments' holdings converged over a number of iterations to a combination that maximized the minimum subset correlation. 


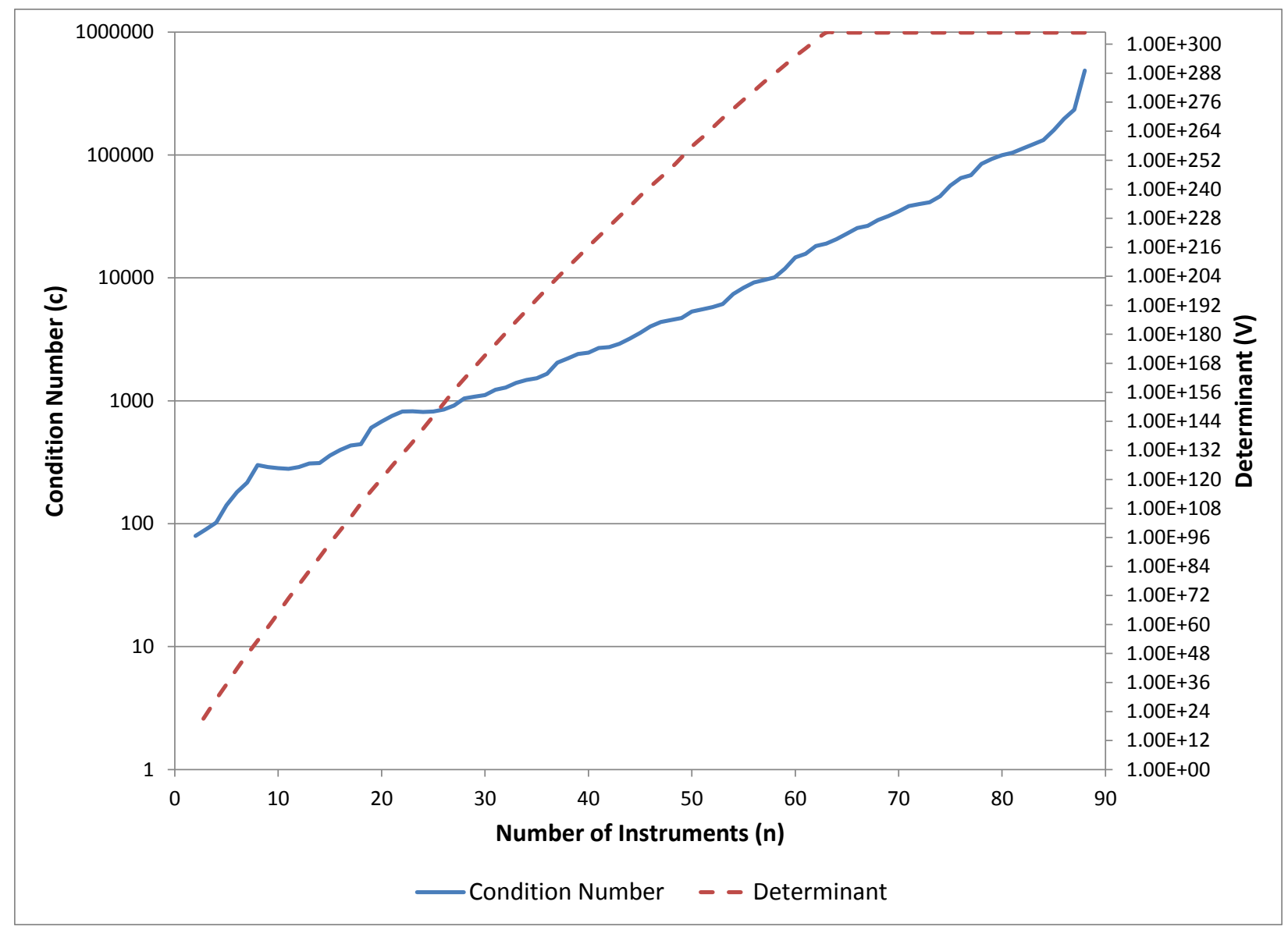

Figure 18 - Reducing the number of instruments (n) by clustering the covariance matrix (V)

This figure illustrates how clustering the covariance matrix improves its numerical condition. We can reduce the number of instruments involved in the MMSC calculation to a value $n$ for which the $N$ subset correlations can be computed within a reasonable timeframe. 


\section{REFERENCES}

- Booth, D. and E. Fama (1992): "Diversification Returns and Asset Contributions", Financial Analyst Journal, 48(3), pp. 26-32.

- Clarke, R., H. de Silva and S. Thorley (2011): "Minimum-Variance Portfolio composition", The Journal of Portfolio Management, Winter.

- Demey, P., S. Maillard and T. Roncalli (2010): "Risk-based indexation", working paper. Available in SSRN: http://ssrn.com/abstract=1582998

- DeMiguel, V., L. Garlappi and R. Uppal (2009): "Optimal versus Naïve Diversification: How inefficient is the 1/N portfolio strategy?", Review of Financial Studies, Vol. 22, pp. 1915-1953.

- Choueifaty, Y. and Y. Coignard (2008): "Toward Maximum Diversification", The Journal of Portfolio Management, 34(4), pp. 40-51.

- Choueifaty, Y., T. Froidure and J. Reynier (2011): "Properties of the Most Diversified Portfolio", working paper. Available in SSRN: http://ssrn.com/abstract=1895459

- Hurst, B., B. Johnson and Y. Ooi (2010): "Understanding Risk Parity”, working paper. AQR Capital.

- Jaeger, L. (2008): “Alternative Beta Strategies and Hedge Fund Replication”, Wiley.

- Litterman, R., and J. Scheinkman (1991): "Common Factors Affecting Bond Returns", Journal of Fixed Income, 1, pp. 62-74.

- Lohre, H., U. Neugebauer and C. Zimmer (2012): "Diversifying Risk Parity”, working paper. Available in SSRN: http://ssrn.com/abstract=1974446

- Lohre, H., H. Opfer and G. Ország, (2012): "Diversified Risk Parity Strategies for Equity Portfolio Selection", working paper. Available in SSRN: http://ssrn.com/abstract=2049280

- López de Prado, M. and D. Leinweber (2012): "Advances in Cointegration and Subset Correlation Hedging Methods”, Journal of Investment Strategies, Vol.1, No. 2 (Spring), pp. 67-115. Available in SSRN: http://ssrn.com/abstract=1906489

- Maillard, S., T. Roncalli and J. Teiletche (2010): "On the properties of equally-weighted risk contribution portfolios", The Journal of Portfolio Management, Vol. 36, No. 4, pp. 60-70.

- Meucci, A. (2009a): "Risk and Asset Allocation", Springer, $3^{\text {rd }}$ Edition.

- Meucci, A. (2009b): "Managing Diversification", Risk, Vol. 22, 74-79.

- Moulton, P. and A. Seydoux (1998): "Using Principal Components Analysis to Structure Buttlerfly Trades", Global Relative Value Research, Deutsche Bank.

- Neukirch, Q. (2008): “Alternative indexing with the MSCI World Index", working paper. Available in SSRN: http://ssrn.com/abstract=1106109

- Scherer, B. (2010): “A new look at Minimum Variance Investing”, working paper. Available at SSRN: http://ssrn.com/abstract=1681306.

- Qian, E. (2005): "Risk parity portfolio: Efficient portfolio through true diversification." Panagora Asset Management, September.

- Qian, E. (2006): "On the financial interpretation of risk contributions: Risk budgets do add up", Journal of Investment Management, Fall. 


\section{DISCLAIMER}

The views expressed in this paper are those of the authors and not necessarily reflect those of Tudor Investment Corporation. No investment decision or particular course of action is recommended by this paper. 